\title{
Inertia Drives a Flocking Phase Transition in Viscous Active Fluids
}

\author{
Rayan Chatterjee, ${ }^{1}$ Navdeep Rana $\odot,{ }^{1}$ R. Aditi Simha, ${ }^{2, *}$ Prasad Perlekar $\odot,{ }^{1}$ and Sriram Ramaswamy $\oplus^{3,1}$ \\ ${ }^{1}$ TIFR Centre for Interdisciplinary Sciences, Tata Institute of Fundamental Research, \\ Gopanpally, Hyderabad 500046, India \\ ${ }^{2}$ Department of Physics, Indian Institute of Technology Madras, Chennai 600 036, India \\ ${ }^{3}$ Centre for Condensed Matter Theory, Department of Physics, Indian Institute of Science, \\ Bangalore 560 012, India
}

(Received 14 January 2021; accepted 28 June 2021; published 21 September 2021)

\begin{abstract}
How fast must an oriented collection of extensile swimmers swim to escape the instability of viscous active suspensions? We show that the answer lies in the dimensionless combination $R=\rho v_{0}^{2} / 2 \sigma_{a}$, where $\rho$ is the suspension mass density, $v_{0}$ the swim speed, and $\sigma_{a}$ the active stress. Linear stability analysis shows that, for small $R$, disturbances grow at a rate linear in their wave number $q$ and that the dominant instability mode involves twist. The resulting steady state in our numerical studies is isotropic hedgehog-defect turbulence. Past a first threshold $R$ of order unity, we find a slower growth rate, of $O\left(q^{2}\right)$; the numerically observed steady state is phase turbulent: noisy but aligned on average. We present numerical evidence in three and two dimensions that this inertia-driven flocking transition is continuous, with a correlation length that grows on approaching the transition. For much larger $R$, we find an aligned state linearly stable to perturbations at all $q$. Our predictions should be testable in suspensions of mesoscale swimmers [D. Klotsa, Soft Matter 15, 8946 (2019)].
\end{abstract}

DOI: 10.1103/PhysRevX.11.031063

Subject Areas: Biological Physics, Fluid Dynamics, Soft Matter

\section{INTRODUCTION}

The theory of active matter [1-9]—-systems whose constituents convert a sustained supply of fuel into movement-is the framework of choice for understanding the collective behavior of motile particles. Like condensed matter in general, active systems display many types of order and operate in a variety of dynamical regimes. Our interest in this paper is in groups of motile organisms in a bulk fluid medium, spontaneously organized into a flock in which their tail-to-head vectors on average point in a common direction. In now-standard terminology [2], we consider polar, wet active matter, described by a vector order parameter characterizing the degree and direction of common orientation and movement.

In the world of Stokesian hydrodynamics, where inertia is absent and viscosity holds sway, an ordered flock in bulk fluid is impossible [10]. Indeed, this limit shapes the defining image of active suspensions as inescapably unstable, dissolving via spontaneous flow $[10,11]$ and defect

\section{*Deceased.}

Published by the American Physical Society under the terms of the Creative Commons Attribution 4.0 International license. Further distribution of this work must maintain attribution to the author(s) and the published article's title, journal citation, and DOI. proliferation [12-27] into a kind of turbulence [19,28-37]. We remind the reader of the instability mechanism $[1,2,10]$ : An aligned state of active particles is a state of uniform uniaxial stress; perturbing this state-through bend or splay, respectively, for "pusher" or "puller" particlescreates spatially varying stresses; force balance requires that these are accompanied by flow; this flow rotates the alignment further in the direction of the perturbation. Note that this description refers neither to the directed motion of the particles nor to acceleration. Stresses and flows are uniaxial but apolar, that is, fore-aft symmetric, and flow responds instantaneously to active stress in the Stokesian approximation. Improved descriptions including polar order alone [10,15,16,38] or inertia alone [10] do not mitigate the instability.

Stable flocks in bulk fluid are, of course, widely observed in the form of fish schools $[39,40]$, which are very polar and very far from Stokesian. We do not venture into the regime of schooling at high Reynolds number, governed by purely inviscid hydrodynamic interactions [41,42], but consider weak inertial effects, which are know to alter significantly the viscous hydrodynamic interaction between slow swimmers [43,44]. A recent perspective [45] makes a persuasive case for the study of active fluids with small but non-negligible inertia, the regime we explore here. A result from Ref. [10] is relevant in this context: A linearized treatment retaining only acceleration and active 
stresses finds a parameter domain in which flocks in fluid are neutrally stable to first order in wave number, with a wavelike dynamic response. Interestingly, such waves of bend excitations have an analog in models without momentum conservation, i.e., "dry" flocking models, when rotational inertia is taken into account [46-49]; the coupled dynamics of classical spin angular momentum and orientation in such models is formally similar to that of hydrodynamic vorticity and orientation in Ref. [10] and the present work. Staying close to viscous hydrodynamics and the force-dipole picture of swimmers, we ask: Can inertia and polar order together defeat the Stokesian instability of flocks?

\section{A. Summary of results}

In this article, focusing on extensile or "pusher" [50] suspensions, we show that the introduction of inertia qualitatively alters our understanding of the viscous hydrodynamics of polar active matter, that is, flocking in fluids. Here are our main results. We show that speed matters: The dimensionless combination $R \equiv \rho v_{0}^{2} / 2 \sigma_{a}$, where $\rho$ is the suspension mass density, $\sigma_{a}$ the scale of active stress, and $v_{0}$ the self-advection speed, governs the stability of active suspensions. Flocks in fluid are stable for large $R$, and their



FIG. 1. $\quad R-\beta$ phase diagram of a polar active suspension. The lines at $R=R_{1}$ and $R=R_{2}$, obtained from linear stability analysis, mark the phase boundaries. For $R<R_{1}$, an aligned state is $O(q)$ unstable, which leads to statistically stationary defect turbulence. In general, a parameter range $\left(R_{1}<R<R_{2}\right)$ where perturbations with small wave number $q$ grow at a rate of $O\left(q^{2}\right)$ intervenes between the stable regime $\left(R>R_{2}\right)$ and the highly unstable regime of $O(q)$ growth but is squeezed out of existence if $\beta=1$; that is, orientation and vorticity have identical diffusivities. The red stars and blue pentagons mark the $(R, \beta)$ values used in our DNS. Insets: order parameter vector field for defect turbulence $\left(R<R_{1}\right)$ with asters (blue dot) and saddles (red square), phase turbulence with orientational order $\left(R_{1}<R<R_{2}\right)$, and a quiescent linearly stable state of complete alignment $\left(R>R_{2}\right)$. inexorable Stokesian instability [10] is the $R=0$ limit of a far richer picture (Fig. 1). For small $R$, perturbations about the aligned state grow at a rate $\propto q$ for wave number $q \rightarrow 0$, while, for moderate $R$, the linear instability persists but with a growth rate $\propto q^{2}$. Crucially, direct numerical simulations of the hydrodynamic equations reveal that the two regimes correspond to qualitatively distinct statistical steady states separated by a nonequilibrium phase transition. The small$R$ regime is isotropic hedgehog-defect turbulence, while that at moderate $R$ is a phase-turbulent [51-54] but ordered flock. Our numerical results suggest a continuous orderparameter onset and a growing correlation length upon approaching the transition.

This paper is organized as follows. In Sec. II, we present the equations of hydrodynamics for polar active suspensions and investigate the linear stability of the uniaxially ordered state. Section III describes our numerical studies, the flocking transition from defect to phase turbulence, and the properties of the turbulent states. We close in Sec. IV with a summary, suggestions for experiment, and open questions.

\section{GOVERNING EQUATIONS AND STABILITY ANALYSIS}

\section{A. Hydrodynamics of active suspensions}

We begin by constructing, from general principles, the hydrodynamic equations of motion for a flock in fluid. We do not employ the language of forces and fluxes or display the dependence of "active" coefficients on a maintained chemical driving force [2,5,55]. We adopt the general, symmetry-based approach of Ref. [10], but our treatment is self-contained and does not presuppose familiarity with that work. The reader sees the results of Ref. [10] emerge as a limiting case in Sec. II B 2. We emphasize that our equations constitute a general effective description on length scales much larger than a swimmer (as we call our self-propelled particles hereafter). They contain parameters such as viscosity and elastic constants; these are phenomenological coefficients in our coarse-grained description of this internally driven system and are named based on the form of the terms they govern. Their values cannot, in principle, be estimated from a near-equilibrium hydrodynamic theory of the suspension. For example, we imagine the viscosity in our equations receives "eddy" contributions from flows on scales of a few swimmers, and we expect that the elastic constants encoding the aligning tendency are at least partly behavioral rather than mechanical. Provided interactions are local in space and time, these features do not limit the validity of our approach, which depends only on conservation laws and symmetries.

For a steady state such as a flock, which spontaneously breaks a continuous invariance, the slow or hydrodynamic variables [56] are the local densities of conserved quantities and the broken-symmetry or Nambu-Goldstone [57,58] 
fields. At a continuous transition to such an ordered state, the amplitude of the order parameter is an additional slow variable. In the absence of reproduction, death, and external forces, the conserved quantities are the total number of swimmers, the total amount of fluid, and the total momentum of swimmers plus fluid. Energy conservation does not play a role, as each swimmer is endowed with a built-in power source. The slow variables corresponding to these conservation laws are then the densities $\rho$ and $\boldsymbol{g}=\rho \boldsymbol{u}$ of mass and momentum of swimmers plus fluid (defining the suspension velocity field $\boldsymbol{u}$ ), respectively, and the number density $c$ of swimmers. The broken-symmetry modes and the magnitude of order are jointly contained in the polar order parameter field $\boldsymbol{p}$, which is the local average of the orientation unit vectors of the particles [10]. It is interesting to note that an equilibrium liquid crystal with macroscopic vectorial, i.e., polar, order has only very recently been discovered [59].

As the order parameter is a space vector, our description is invariant under the joint inversion of $\boldsymbol{p}$ and the spatial coordinate $\boldsymbol{r}$, but not $\boldsymbol{p}$ alone. The absence of $\boldsymbol{p} \rightarrow-\boldsymbol{p}$ symmetry is central to our narrative. We, therefore, include at this stage, at leading order in gradients, all terms that break this symmetry in the equations of motion $[60,61]$ (see also Refs. [2,15,16,62-64]), although we shortly pass to a more economical description. The equations read

$\rho\left(\partial_{t} \boldsymbol{u}+\boldsymbol{u} \cdot \nabla \boldsymbol{u}\right)=-\nabla P+\mu \nabla^{2} \boldsymbol{u}+\nabla \cdot\left(\boldsymbol{\Sigma}^{a}+\boldsymbol{\Sigma}^{r}\right)$,

$\partial_{t} \boldsymbol{p}+\left(\boldsymbol{u}+v_{0} \boldsymbol{p}\right) \cdot \nabla \boldsymbol{p}=\lambda \boldsymbol{S} \cdot \boldsymbol{p}+\boldsymbol{\Omega} \cdot \boldsymbol{p}+\Gamma \boldsymbol{h}+\ell \nabla^{2} \boldsymbol{u}$,

and

$$
\partial_{t} c+\nabla \cdot\left[\left(\boldsymbol{u}+v_{1} \boldsymbol{p}\right) c\right]=0 .
$$

Equation (3) expresses number conservation; the active particles self-propel with velocity $v_{1} \boldsymbol{p}$ in the frame of the suspension and, hence, $\boldsymbol{u}+v_{1} \boldsymbol{p}$ in the laboratory frame. In Eq. II A, the polar order parameter $\boldsymbol{p}$ is carried by the hydrodynamic velocity $\boldsymbol{u}$ and by its self-advection $v_{0}$ [not related by any symmetry $[49,65]$ to $v_{1}$ in Eq. (3)], $\boldsymbol{S}$ and $\boldsymbol{\Omega}$ are the symmetric and antisymmetric parts, respectively, of the velocity gradient tensor $\nabla \boldsymbol{u}$ which couple orientation to flow as in ordinary nematic liquid crystals [66], $\Gamma$ is the kinetic coefficient governing relaxation in the local molecular field $\boldsymbol{h}$ to be discussed below, and $\ell$ is the polar flow coupling at leading order in a gradient expansion. The $v_{0}, v_{1}$, and $\ell$ terms are polar: Their presence implies that the equations are not invariant under $\boldsymbol{p} \rightarrow-\boldsymbol{p}$. In Eq. (1), the hydrodynamic pressure $P$ enforces incompressibility $\nabla \cdot \boldsymbol{u}=0$.

$$
\boldsymbol{\Sigma}^{a} \equiv-\sigma_{a} \boldsymbol{p p}-\gamma_{a}\left(\nabla \boldsymbol{p}+\nabla \boldsymbol{p}^{T}\right)
$$

is the intrinsic stress associated with swimming activity, which we display up to first subleading order in gradients. In Eq. (4), $\sigma_{a}$ is the force-dipole density $[2,10,67]$. In microscopic terms, the forces exerted by a swimmer and the ambient fluid on each other add to zero, so the associated force density has zero monopole moment. The minimal model for a swimmer is, thus, a point dipolar force density. A collection of such swimmers, each with dipole strength $W$, local concentration $c$, and mean local alignment given by the polar order parameter $\boldsymbol{p}$, can readily be seen $[2,10,67]$ to have force density $-W \nabla \cdot(c \boldsymbol{p p}) . W>0$ and $W<0$ correspond, respectively, to extensile swimmers, that push fluid back with their tails and move forward, and contractile swimmers, that advance by pulling fluid toward themselves from the front. Thus, in Eq. (4), $\sigma_{a}=W c$. The polar contribution to the active stress, given at leading order in a gradient expansion by the $\gamma_{a}$ term in Eq. (4), arises $[62,67]$ if the force dipole on each particle is displaced with respect to the center of drag of the particle, as it must be to achieve locomotion. From the foregoing, it is plausible that $\gamma_{a}$ should be proportional to $\sigma_{a}$, with the proportionality factor being a length that measures the fore-aft asymmetry of the active particles. In principle, all parameters in our equations should be functions of the local concentration $c$. Phenomenologically, $\sigma_{a}$ and $\gamma_{a}$ are tied to the presence of active particles and should, therefore, be proportional to $c$ for $c \rightarrow 0$. In the simple microscopic picture discussed above, if $W$ is treated as an intrinsic single-particle property and, therefore, independent of $c$, the proportionality is exact. The contribution

$$
\boldsymbol{\Sigma}^{r}=\frac{1-\lambda}{2} \boldsymbol{p h}-\frac{1+\lambda}{2} \boldsymbol{h} \boldsymbol{p}-\ell\left(\nabla \boldsymbol{h}+\nabla \boldsymbol{h}^{T}\right)
$$

is the reversible thermodynamic stress for an equilibrium polar liquid crystal. The expression (5) extends the form found in Refs. $[2,68]$ to include the leading-order polar $\ell$ term, which is the Onsager counterpart of the polar flowcoupling term $\ell \nabla^{2} \boldsymbol{u}$ in Eq. II A, discussed in Refs. [60,61]. $\boldsymbol{h}=-\delta F / \delta \boldsymbol{p}$ is the molecular field conjugate to $\boldsymbol{p}$, derived from a free-energy functional

$$
F=\int d^{3} r\left[\frac{1}{4}(\boldsymbol{p} \cdot \boldsymbol{p}-1)^{2}+\frac{K}{2}(\nabla \boldsymbol{p})^{2}-E \boldsymbol{p} \cdot \nabla c\right]
$$

favoring a $\boldsymbol{p}$ field of uniform magnitude [69] which we rescale to unity. A single Frank constant [70-72] $K$ penalizes gradients in $\boldsymbol{p}$, and $E$ promotes alignment of $\boldsymbol{p}$ up or down gradients of $c$, according to its sign. $\mu$ is the shear viscosity of the suspension, and $\Gamma$, the collective rotational mobility for the relaxation of the polar order parameter field, is expected to be of the order of $1 / \mu$. $\lambda$ is the nematic flow-alignment parameter [73,74], and $\ell$, with units of length, governs the lowest-order polar 
flow-coupling term [60,61]. If our equations were derived from a microscopic model of particles in a fluid, we expect that both $\ell$ and the length $\gamma_{a} / \sigma_{a}$ would be related to a foreaft asymmetry in the dimensions of the active particles.

\section{Essential and incidental polar contributions}

Equations (1)-(3) are endowed with a surfeit of parameters originating in the polar character of our system - the speeds $v_{0}$ and $v_{1}$ at which the orientation advects itself and the concentration, respectively, the polar active stress coefficient $\gamma_{a}$ and the passive polar flow-coupling length scale $\ell$. In the work [10] that initiated the study of the hydrodynamics of active liquid crystals, the polar character of the order parameter of a flock plays an important role, combining with inertia to yield a propagative mode structure. As this structure is a leading-order feature in a gradient expansion, neither $\gamma_{a}$ nor $\ell$, which enter at next-toleading order relative to $\sigma_{a}$ in Eq. (4) and $1 \pm \lambda$ in Eqs. (5) and II A, respectively, were considered, and polar effects thus enter [10] only through $v_{0}$ and $v_{1}$. The instability of active liquid crystals in the Stokesian domain - the other major finding of Ref. [10]— commands much greater interest in the field thanks to its connection to experimental realizations in cellular and microbial settings. An analysis that ignores polarity altogether and works only with the axis of orientation offers a satisfactory conceptual understanding of that instability [2,30], though interesting complexities arise [15] in a Stokesian setting through the polar parameters $\gamma_{a}, v_{0}$, and $v_{1}$. A final remark in this context is that polarity asserts its presence in any formulation in terms of a vector order parameter $\boldsymbol{p}$, even if the equations of motion are invariant under $\boldsymbol{p} \rightarrow-\boldsymbol{p}$, through the nature of topological defects $[20,75]$.

For the purposes of the present work, what matters is that the self-advection speed $v_{0}$ plays a distinct-and crucialrole. The other polar parameters contribute in an incidental manner. First, we are concerned here only with the extensile case $\sigma_{a}>0$, for which the instability mode is bend, which decouples from concentration in the linear theory. In what follows, we therefore ignore the concentration field, and, hence, $v_{1}$ drops out of our analysis. Next, as we show in the Appendix B, it is only through $v_{0}$-in the form $\rho v_{0}^{2}$ and its competition with $\sigma_{a}$-that the stabilizing effects of inertia enter our treatment. $\gamma_{a}$ and $\ell$ leave unaltered both the coefficient of the $O(q)$ contribution to the mode frequency and the parameter value at which the instability growth rate changes from $O(q)$ to $O\left(q^{2}\right)$. They simply shift the coefficients of the $O\left(q^{2}\right)$ piece of the mode frequency by amounts of relative order unity. We, therefore, work with an economical description in which $\gamma_{a}$ and $\ell$ are zero and polar effects enter only through $v_{0}$ and, of course, the nature of the allowed topological defects. Crucially, $v_{0}$ and $\sigma_{a}$ are independent quantities in our coarse-grained treatment, a point we return to later in the paper.

\section{B. Linear stability analysis}

Defining the ordering direction to be $\hat{\boldsymbol{x}}$ and directions in the $y z$ plane as $\perp$, we investigate the stability of a uniform ordered flock $\left(c=c_{0}, \boldsymbol{u}=\mathbf{0}\right.$, and $\left.\boldsymbol{p}=\hat{\boldsymbol{x}}\right)$, which is a stationary solution of Eqs. (1)-(3) to small perturbations $\left(\delta \boldsymbol{u}_{\perp}, \delta \boldsymbol{p}_{\perp}, \delta c\right)$, where the presence of only the $\perp$ components is a result of incompressibility and the "fast" nature of $p_{x}$. We present here the results for the case where the concentration field $c$ is removed from the analysis. This case is sufficient for our purposes, because $c$ does not participate significantly in the linear instabilities of relevance, as we now argue. Taking the curl with respect to $\nabla_{\perp}$ eliminates $c$ from the $\perp$ component of Eq. (II A). A similar curl removes it from the $\perp$ component of Eq. (1) as well. Thus, concentration does not participate in the linear dynamics of the twist-bend mode [10]. The threedimensional instability of extensile active fluids is known, numerically, to be twist dominated [76], an observation for which our linear stability analysis below provides the natural explanation. A description without a concentration field should, thus, be a reasonable guide to instabilities and active turbulence in our system. It is important to note that the neglect of the concentration field in our treatment does not amount to an incompressibility constraint on the polar order parameter field. A formal connection between the complete equations and those without a concentration field can be achieved by introducing birth and death of particles so that $c$ becomes "fast" [77] and can be eliminated in favor of the slow variables $\boldsymbol{p}_{\perp}$ and $\boldsymbol{u}_{\perp}$, with at most a finite shift in parameter values in the equations for the slow variables. Changes in the linear stability analysis upon inclusion of the concentration are quantitative, not qualitative, and can be found in the Appendix A. Defining the projector

$$
\mathbf{T}_{q} \equiv \mathbf{I}-\hat{\boldsymbol{q}} \hat{\boldsymbol{q}}
$$

transverse to $\boldsymbol{q}$ and linearizing Eqs. (1) and II A about the ordered state, we find

$$
\begin{aligned}
\left(\rho \partial_{t}+\mu q^{2}\right) \delta \boldsymbol{u}_{\perp \boldsymbol{q}}= & -i \mathbf{T}_{\boldsymbol{q}} \cdot\left[\left(\sigma_{a}+\frac{\lambda-1}{2} K q^{2}\right) \hat{\boldsymbol{x}} \boldsymbol{q}_{\perp}\right. \\
& \left.+q_{x}\left(\sigma_{a}+\frac{\lambda+1}{2} K q^{2}\right) \mathbf{I}\right] \cdot \delta \boldsymbol{p}_{\perp \boldsymbol{q}}, \\
\partial_{t} \delta \boldsymbol{p}_{\perp \boldsymbol{q}}= & +i\left(\frac{\lambda+1}{2} q_{x} \mathbf{I}-\frac{\lambda-1}{2} \frac{\boldsymbol{q}_{\perp} \boldsymbol{q}_{\perp}}{q_{x}}\right) \cdot \delta \boldsymbol{u}_{\perp \boldsymbol{q}} \\
& -\left(i v_{0} q_{x}+\Gamma K q^{2}\right) \delta \boldsymbol{p}_{\perp \boldsymbol{q}} .
\end{aligned}
$$

As in Ref. [10], the divergence and curl of Eqs. (8) and (9) describe, respectively, the dynamics of splay and twist, with an admixture of bend in each case for $q_{x} \neq 0$. Defining $\phi$ to be the angle between the wave vector $\boldsymbol{q}$ and the alignment $(\hat{\boldsymbol{x}})$ direction, the resulting dispersion 
relations for the frequency $\omega$, valid for all $\boldsymbol{q}$, for modes of the form $e^{i(\boldsymbol{q} \cdot \boldsymbol{r}-\omega t)}$, are

$$
\begin{aligned}
\omega=\omega_{ \pm}^{s}= & \frac{1}{2} v_{0} q \cos \phi-i \frac{\mu_{+}}{2 \rho} q^{2} \pm\left(\frac{\sigma_{a}}{2 \rho}\right)^{1 / 2} \\
& \times\left[A(\phi) q^{2}+i B(\phi) q^{3}+G(\phi) q^{4}\right]^{1 / 2}
\end{aligned}
$$

for the splay-bend modes and

$$
\begin{aligned}
\omega_{ \pm}^{t}= & \frac{1}{2} v_{0} q \cos \phi-i \frac{\mu_{+}}{2 \rho} q^{2} \pm\left(\frac{\sigma_{a}}{2 \rho}\right)^{1 / 2} \\
& \times\left[A(0) \cos ^{2} \phi q^{2}+i B(0) \cos \phi q^{3}+\bar{G}(\phi) q^{4}\right]^{1 / 2}
\end{aligned}
$$

for the twist-bend modes. In Eqs. (10) and (11), we define $A(\phi) \equiv R \cos ^{2} \phi-\cos 2 \phi(1+\lambda \cos 2 \phi), \quad B(\phi) \equiv$ $\left(v_{0} \mu_{-} / \sigma_{a}\right) \cos \phi, \quad G(\phi)=-\left(\mu_{-}^{2} / 2 \rho \sigma_{a}\right)+\left(K / 2 \sigma_{a}\right)(1+$ $\lambda \cos 2 \phi)^{2}$, and $\bar{G}(\phi) \equiv-\left(\mu_{-}^{2} / 2 \rho \sigma_{a}\right)+\left(K / 2 \sigma_{a}\right)(1+$ $\lambda)^{2} \cos ^{2} \phi[78]$ with

$$
R \equiv \rho v_{0}^{2} / 2 \sigma_{a},
$$

and $\mu_{ \pm} \equiv \mu(1 \pm \beta)$, where $\beta \equiv \Gamma K \rho / \mu$ should be of the same order as $\alpha \equiv K \rho / \mu^{2}$ because the mobility $\Gamma \sim 1 / \mu$. For conventional liquid crystals $\alpha, \beta \ll 1$.

When $R=0$, the extensile $\left(\sigma_{a}>0\right)$ systems of interest here present a bend instability [see Eqs. (10) and (11)] with invasion speed $\sqrt{\sigma_{a} / \rho}$. For $v_{0}>0$, disturbances can outrun this invasive growth. The dimensionless combination $R$ describes this competition. Note that the contribution of $R$ vanishes for pure splay, Eq. (10) at $\phi=\pi / 2$, so motility cannot stabilize contractile $\left(\sigma_{a}<0\right)$ flocks in fluid.

\section{Small-q behavior: The $O(q)$ and $O\left(q^{2}\right)$ instabilities}

Let us first examine the small- $q$ behavior. Expanding Eqs. (10) and (11) up to order $q^{2}$, we then find

$$
\begin{aligned}
\omega=\omega_{ \pm}^{s}= & \frac{q}{2}\left\{v_{0} \cos \phi \pm\left[\frac{2 \sigma_{a}}{\rho} A(\phi)\right]^{1 / 2}\right\} \\
& -\frac{i}{2} \frac{\mu}{\rho} q^{2}\left\{1+\beta \mp(1-\beta)\left[\frac{R \cos ^{2} \phi}{A(\phi)}\right]^{1 / 2}\right\}
\end{aligned}
$$

for the splay-bend modes and

$$
\begin{aligned}
\omega=\omega_{ \pm}^{t}= & \frac{q}{2} \cos \phi\left\{v_{0} \pm\left[\frac{2 \sigma_{a}}{\rho} A(0)\right]^{1 / 2}\right\} \\
& -\frac{i}{2} \frac{\mu}{\rho} q^{2}\left\{1+\beta \mp(1-\beta)\left[\frac{R}{A(0)}\right]^{1 / 2}\right\}
\end{aligned}
$$

for the twist-bend modes. Here, $A(0)=A(\phi=0)=$ $R-(1+\lambda)$. One note of caution: The small $q$ expansion that leads to Eq. (14) assumes $v_{0} q \cos \phi>q^{2} \mu / \rho$, which means that it does not apply for $\phi=\pi / 2$, i.e., pure twist.
It does, however, hold for any $\phi \in[0, \pi / 2)$, but the closer $\phi$ is to $\pi / 2$, the smaller $q$ must be for the result to apply.

Two of our main results now follow. If $R<1+\lambda$, Eq. (14) signals a bend instability with small- $q$ growth rate $\sim q$. This result is discussed in the strictly apolar case $v_{0}=0$ in Ref. [10] and can be viewed as the small- $q$ extension of the Stokesian bend instability [10]. However, if $R>1+\lambda$, so that the $O(q)$ instability is averted, $0<1-(1+\lambda) / R<1$. If $R$ is not too large, this result means the coefficient of $i q^{2}$ in Eqs. (13) and (14) is positive, signaling a small- $q$ instability with diffusive growth. This $O\left(q^{2}\right)$ instability exists for $R$ between $R_{1}=1+\lambda$ and

$$
R_{2}=\frac{\mu_{+}^{2}}{\mu_{+}^{2}-\mu_{-}^{2}} R_{1}=\frac{1+\lambda}{4 \beta}(1+\beta)^{2} .
$$

For $R>R_{2}$, the flock is linearly stable. If $\beta \ll 1$ as in molecular systems, $R_{2} \gg R_{1}$, and the $O\left(q^{2}\right)$ instability occupies a large range of $R$. In the $\beta=0$ limit, the uniformly ordered flock is always linearly unstable, with small- $q$ growth rate $\sim q$ for $R<1+\lambda$ and $\sim q^{2}$ for $R>1+\lambda$. Figure 1 summarizes the small- $q$ stability behavior.

Note that the $O\left(q^{2}\right)$ instability can be eliminated in the special case $\beta=1$, i.e., $\mu / \rho=\Gamma K$. Noting that $\Gamma$ should be roughly $1 / \mu$, this condition implies $K=\mu^{2} / \rho$, an interesting condition that equates a Frank constant (which, recall, has units of force in three dimensions) to Purcell's intrinsic force scale [79] $\mu^{2} / \rho$ for three-dimensional viscous fluids. As we remark above, $\beta$ in molecular or colloidal systems is about $10^{-4}[66,80]$, so requiring it to be of the order of unity amounts to insisting that the swimmers have an exceptionally strong aligning interaction. This possibility cannot be ruled out a priori, as alignment in living systems is likely to be active and behavioral, not a passive mechanical torque.

\section{Large-q dynamics and the Stokesian limit}

Having established the general linearized behavior of active extensile liquid crystals in the true hydrodynamic regime of small wave numbers, we turn our attention to large wave numbers. Two length scales are important here:

$$
\ell_{v} \equiv \mu / v_{0} \rho \quad \text { and } \quad \ell_{\sigma} \equiv \mu / \sqrt{\rho \sigma_{a}}=R^{1 / 2} \ell_{v},
$$

below which viscosity overwhelms the inertial effects of self-advection, and

$$
\ell_{K} \equiv \sqrt{K / \sigma_{a}}
$$

below which Frank elasticity dominates active stresses. For molecular or colloidal systems, for which, as we remark earlier, $\alpha$ is exceedingly small $[66,80], \ell_{K} / \ell_{v}=\sqrt{\alpha R}$ 
should be small too except in the unlikely condition of ultrahigh self-advection speeds. The wave number range $\max \left(\ell_{v}^{-1}, \ell_{\sigma}^{-1}\right) \ll q \ll \ell_{K}^{-1}$ should be substantial. Expanding Eqs. (10) and (11) for $q \gg \max \left(\ell_{v}^{-1}, \ell_{\sigma}^{-1}\right)$, we find, to leading order in $\alpha$ and $\beta$, that the splay-bend mode that goes unstable at small $R$ has the form

$$
\begin{aligned}
\omega^{s}= & -i \frac{\sigma_{a}}{2 \mu} A(\phi)+v_{0} q \cos \phi \\
& -i\left[\Gamma \mu+\frac{1}{4}(1+\lambda \cos 2 \phi)^{2}\right] \frac{K}{\mu} q^{2} \\
= & -\frac{i}{2 \mu}\left[\frac{\rho v_{0}^{2}}{2} \cos ^{2} \phi-\sigma_{a} \cos 2 \phi(1+\lambda \cos 2 \phi)\right] \\
& +O\left(q,-i q^{2}\right)
\end{aligned}
$$

and the corresponding twist-bend mode has frequency

$$
\begin{aligned}
\omega^{t}= & -i \frac{\sigma_{a}}{2 \mu} A(0) \cos ^{2} \phi+v_{0} q \cos \phi \\
& -i\left[\Gamma \mu+\frac{1}{4}(1+\lambda)^{2} \cos ^{2} \phi\right] \frac{K}{\mu} q^{2} \\
= & -\frac{i}{2 \mu}\left[\frac{\rho v_{0}^{2}}{2}-(1+\lambda) \sigma_{a}\right] \cos ^{2} \phi+O\left(q,-i q^{2}\right),
\end{aligned}
$$

where $A(\phi)$ is as defined in Eqs. (10) and (11). The Stokesian instability of active liquid crystals $[2,10,11]$, with a single growth-rate scale $\sigma_{a} / \mu$, emerges from Eqs. (18) and (19) if $\rho$ is set to zero. For $\sigma_{a}<0$, contractile or pusher suspensions, which is not the case we are focusing on in this work, Eq. (18) predicts an instability for $\phi>\pi / 4$, which is splay dominated. For $\sigma_{a}>0$, the extensile or pusher case, Eq. (18) predicts an instability for $\phi<\pi / 4$, which is bend dominated. More importantly, Eq. (19) predicts an bend instability for all directions other than pure twist $\phi=\pi / 2$, although, of course, the value of $q$ below which the instability is seen approaches 0 as $\phi \rightarrow \pi / 2$. In general, however, Eqs. (18) and (19) are not Stokesian expressions but short-wavelength limits of the linearized dynamics of a polar active suspension with inertia, which enters through $R$. We see, in particular, that the stability criteria in this large- $q$ regime are identical to those for the $O(q)$ mode at small $q$. Thus, a twist-bend instability, with a growth rate $\sim \sigma_{a} / \mu$ for $\max \left(\ell_{v}^{-1}, \ell_{\sigma}^{-1}\right) \ll q \ll \ell_{K}^{-1}$, takes place if $R<1+\lambda$. This result establishes our claim that the $O(q)$ instability is the small- $q$ extension of the Stokesian instability [10] of active suspensions. The $O\left(q^{2}\right)$ instability that intervenes at small $q$ as $R$ is increased does not reflect itself in the large- $q$ dynamics. Figure 2 displays the growth or decay rates of the twist-bend mode as a function of the wave number as $R$ is varied at $\beta=10^{-4}$.

It is important to keep in mind that the active stress $\sigma_{a}$ is a partial description of the mechanics of self-propulsion

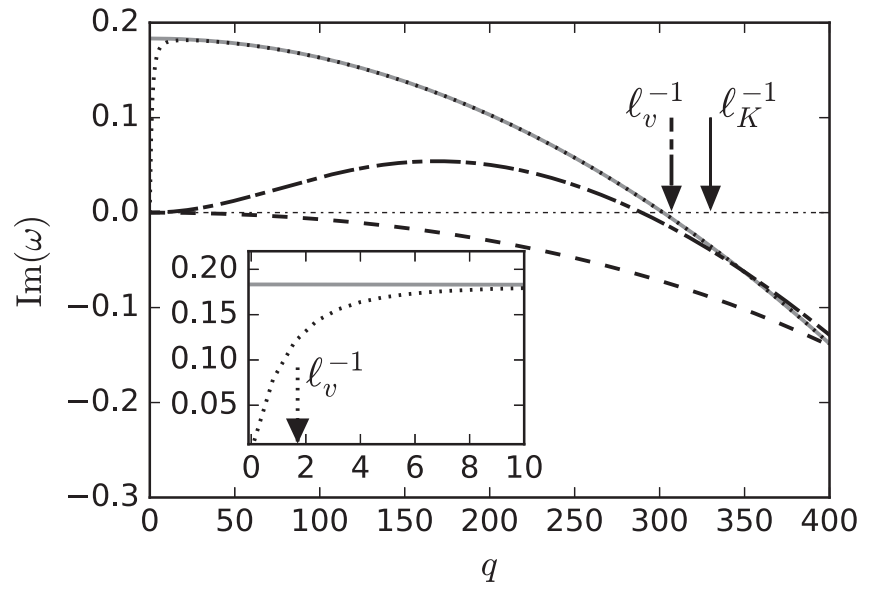

FIG. 2. Growth rate versus wave number. Gray line, Stokesian limit; black dotted line, $O(q)$ unstable, $\ell_{v}=1$, and $R=5 \times 10^{-2}$; black dash-dotted line, $O\left(q^{2}\right)$ unstable, $\ell_{v}=3 \times 10^{-3}$, and $R=4.5 \times 10^{3}$; black dashed line, stable, $\ell_{v}=3 \times 10^{-4}$, and $R=4.5 \times 10^{5}$. Arrows indicate the wave number corresponding to $\ell_{v}$ for the unstable cases. For all the dispersion curves, we use $\phi=55^{\circ}$ and $K=10^{-6}$, which sets $\ell_{K}=3.2 \times 10^{-3}$.

based on an estimate of the force-dipole concentration and is not a priori determined by $v_{0}$. To take an extreme case, Stokesian swimmers with no force dipole exist, e.g., the pure quadrupole $[79,81,82]$. Assuming a volume fraction of the order of unity, let us nonetheless try to estimate $R$ for typical swimmers of speed $v_{0}$ (the distinction between the speeds of self-propulsion and self-advection being unimportant for this discussion) and size $b$ (although we must remember that this size is notional in our coarse-grained description). For Reynolds number Re small at the scale of the individual organism, it is plausible that $\sigma_{a} \sim \mu v_{0} / b$. In that case, $R \equiv \rho v_{0}^{2} / 2 \sigma_{a} \sim \rho v_{0} b / \mu=\operatorname{Re} \ll 1$, so we can replace Eqs. (18) and (19) by their Stokesian approximations. For swimmers at a nonzero Reynolds number, it is less obvious how to estimate $\sigma_{a}$. If we take it still to be a viscous stress, then $R=\operatorname{Re}$ continues to hold, so now $R$ dominates in Eqs. (18) and (19), or in Eqs. (10) and (11), guaranteeing stability. Even if $\sigma_{a} \sim \rho v_{0}^{2}, R \sim 1$ and it is plausible that the instability is averted [83].

Dominance of twist in the three-dimensional extensile instability.-A noteworthy feature, to our knowledge not discussed in the literature, emerges in our threedimensional analysis: There are two families of bend instability-mixed with splay as in Eqs. (13) and (18) and twist as in Eqs. (14) and (19). Interpolation with bend mitigates the instability in Eqs. (13) and (18), crossing over to stability for large enough $\phi$, but twist in Eqs. (14) and (19) has no such effect. The twist-bend instability Eqs. (14) and (19) should thus dominate, as it occurs for all $\phi$ except precisely $\pi / 2$. This abundance of twisted unstable modes in Eqs. (14) and (19), independent of the roles of polarity and 
inertia, is doubtless the explanation of the numerical observations of Shendruk et al. [76] in their study of three-dimensional extensile active nematics.

We summarize this section by noting that, when inertia is taken into account, orientable active suspensions can have two types of linear instability at small wave number $q$, governed by the dimensionless parameter $R$ [Eq. (12)]. The instability growth rates are $O(q)$ for $R<R_{1}=1+\lambda$, where $\lambda$ is a flow-alignment parameter, and $O\left(q^{2}\right)$ for $R_{1}<R<R_{2} \sim R_{1} / \beta$, where $\beta$ is defined in Eqs. (10) and (11). Linearly stable behavior is found for $R>R_{2}$. As $\beta \sim 10^{-4}$ in molecular systems, the $O\left(q^{2}\right)$-unstable regime occupies a rather large range in parameter space. Indeed, one could argue that the typical behavior is that corresponding to the $\beta=0$ limit, in which the aligned state is always linearly unstable, either at $O(q)$ or at $O\left(q^{2}\right)$. In Sec. III, we gain insight beyond this linear analysis through a detailed numerical study to discover the long-time fate of the system in these unstable regimes.

\section{NUMERICAL STUDIES OF ACTIVE HYDRODYNAMICS WITH INERTIA}

In the following section, we describe detailed numerical solutions of our equations, with an emphasis on the changes in behavior as $R$ is varied. We first verify the predictions of our linear stability analysis. Next, for extensile suspensions, we reveal an inertia-driven nonequilibrium phase transition from a disordered defectturbulent state for $0<R<R_{1}$ to an ordered phaseturbulent state for $R_{1} \leq R<R_{2}$. We characterize these using the polar order parameter, that is, the macroscopic steady-state average of $\boldsymbol{p}$, correlation functions, and energy spectra.

\section{A. Direct numerical simulations (DNS)}

We numerically integrate Eqs. (1) and II A in square and cubic domains of volume $\mathcal{L}^{d}$ in dimensions $d=2$ and 3. Spatial discretization, with $N^{d}$ collocation points, is conducted by employing a pseudospectral method [84] for Eq. (1) and a fourth-order central finite-difference scheme for Eq. II A. For temporal integration, we use a second-order Adams-Bashforth scheme [85]. Consistent with the linear stability analysis conducted earlier, we choose a uniform ordered state with transverse monochromatic perturbation as the initial condition, i.e., $\boldsymbol{u}=\mathbf{0}+A \hat{\boldsymbol{e}}_{\perp} \cos \boldsymbol{q} \cdot \boldsymbol{r}, \boldsymbol{p}=\hat{\boldsymbol{x}}+B \hat{\boldsymbol{e}}_{\perp} \cos \boldsymbol{q} \cdot \boldsymbol{r}$, where $\hat{\boldsymbol{e}}_{\perp} \equiv$ $(\hat{\boldsymbol{y}}+\hat{\boldsymbol{z}}) / \sqrt{2}$ is a unit vector in the plane perpendicular to the ordering direction, and we make the arbitrary but acceptable choice $A=B=10^{-3}$.

We monitor the time evolution of perturbations and, in the turbulent steady state, investigate the statistical properties of the velocity and the director fields. In Table I, we summarize the parameters used in our DNS.
TABLE I. Spatial dimension $D$ of the domain and parameters $\mathcal{L}, N, v_{0}, K$, and $R$ used in our direct numerical simulations. The suspension density $\rho=1, \lambda=0.1$, and $\mu=0.1$ and the rotational mobility $\Gamma=1$ are kept fixed for all the runs. Note that as $R$ approaches $R_{2}$ the range of linearly unstable modes shrinks and is restricted to small wave numbers, i.e., large length scales. To resolve these unstable modes as well as the small-scale fluctuations that arise because of the nonlinear couplings, for $R=8$ we use a square domain with each side of length $160 \pi$ and discretize it with $4096^{2}$ collocation points.

\begin{tabular}{lcccccc}
\hline \hline & $\mathrm{D}$ & $\mathcal{L}$ & $N$ & $v_{0}\left(\times 10^{-2}\right)$ & $K\left(\times 10^{-3}\right)$ & $R \equiv \rho v_{0}^{2} / 2 \sigma_{a}$ \\
\hline SPP1 & 3 & $2 \pi$ & 128 & 3.16 & 1 & $0.02,0.0625$ \\
SPP2 & 3 & $10 \pi$ & 160 & $0.7,13.4$ & 2 & $0.01,4$ \\
SPP3 & 3 & $10 \pi$ & 320 & 3.16 & 1 & $0.1-2$ \\
SPP4 & 2 & $20 \pi$ & 1024 & 3.16 & 1 & $0.05-2.0$ \\
SPP5 & 2 & $32 \pi$ & 1024 & 3.16 & 1 & $0.15,0.20$ \\
SPP6 & 2 & $40 \pi$ & 2048 & 3.16 & 1 & $0.25,0.30,0.35$ \\
SPP7 & 2 & $64 \pi$ & 3072 & 3.16 & 1 & $0.4-0.6$ \\
SPP8 & 2 & $80 \pi$ & 4096 & 3.16 & 1 & 0.7 \\
SPP9 & 2 & $80 \pi$ & 2048 & 3.16 & 1 & 1.25 \\
SPP10 & 2 & $160 \pi$ & 4096 & 3.16 & 1 & 8.0 \\
SPP11 & 2 & $128 \pi$ & 8192 & 3.16 & 1 & 0.01 \\
\hline \hline
\end{tabular}

\section{B. Initial growth of instabilities}

We now present a comparison between the short-time growth obtained from the DNS with the analytical predictions of the linear stability analysis. The plot of the bend-twist dispersion curve given by Eq. (11) for $\phi=55^{\circ}$ is shown in Fig. 3. The black dots indicate the initial temporal growth rate of perturbations obtained from our DNS, which shows excellent agreement with the analytical



FIG. 3. Comparison of the growth rates obtained from dispersion relation Eq. (11) with those from DNS (black dots). Inset: initial time evolution of the perturbation amplitude $\left|\boldsymbol{q}_{\perp} \times \delta \boldsymbol{p}_{\perp q}\right|$ for $O(q): R=10^{-2}$ (a) and $O\left(q^{2}\right): R=4$ (b) growth rates (run SPP2). Note that we choose $\phi=55^{\circ}$ for the initial perturbations. 
results. Furthermore, our simulations correctly capture the exponential and oscillatory characters of the growth for $R<R_{1}$ and $R_{1}<R<R_{2}$, respectively. Note that, for $R<R_{1}$, the exponential growth rate of perturbations is much faster than the oscillatory kinematic contribution $\operatorname{Re}[\omega]=v_{0} q \cos \phi$. For $R_{1}<R<R_{2}, \operatorname{Re}[\omega]$ has contributions from both the kinematic and the inertial terms. Therefore, we observe an exponential growth of $\left|\boldsymbol{q}_{\perp} \times \boldsymbol{\delta} \boldsymbol{p}_{\perp \boldsymbol{q}}\right|$ for $R<R_{1}$ [see Fig. 3(a)] but oscillatory growth for $R_{1}<R<R_{2}$ [see Fig. 3(b)].

\section{A flocking phase transition}

We now investigate the morphology and statistical properties of the orientation and flow emerging from the instabilities discussed above. Figure 4 shows the typical flow structures observed in our DNS with increasing $R$ in the statistically steady state. For $0<R<R_{1}$, we observe hedgehog defects. The interdefect spacing grows with increasing $R$. Unexpectedly, when $R$ increases past the first threshold $R_{1}$, a fluctuating but on average aligned state emerges. As we remarked in the introduction, this state is clear numerical evidence that $R=R_{1}$ marks a nonequilibrium phase transition from a statistically isotropic state to a flock or, in the terminology of spatiotemporal chaos, from defect turbulence to phase turbulence $[51,86,87]$. In the latter state, long-wavelength statistical variation of the broken-symmetry variable is present, but the amplitude of the order parameter is not destroyed by defects. We do not, however, measure the system-size

\section{Phase turbulence Stable flock}

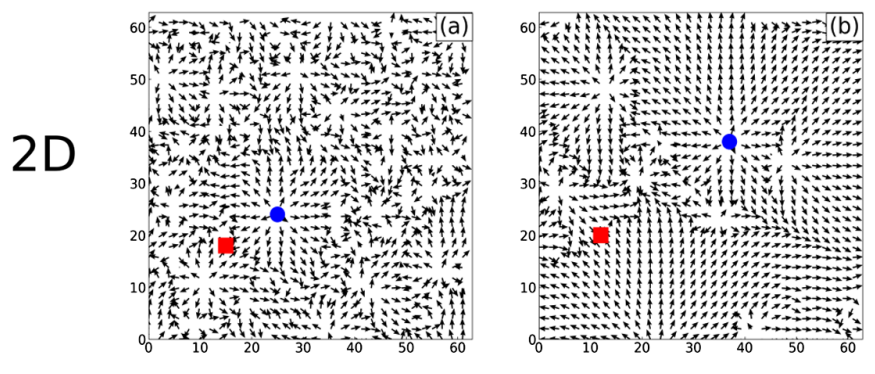

3D
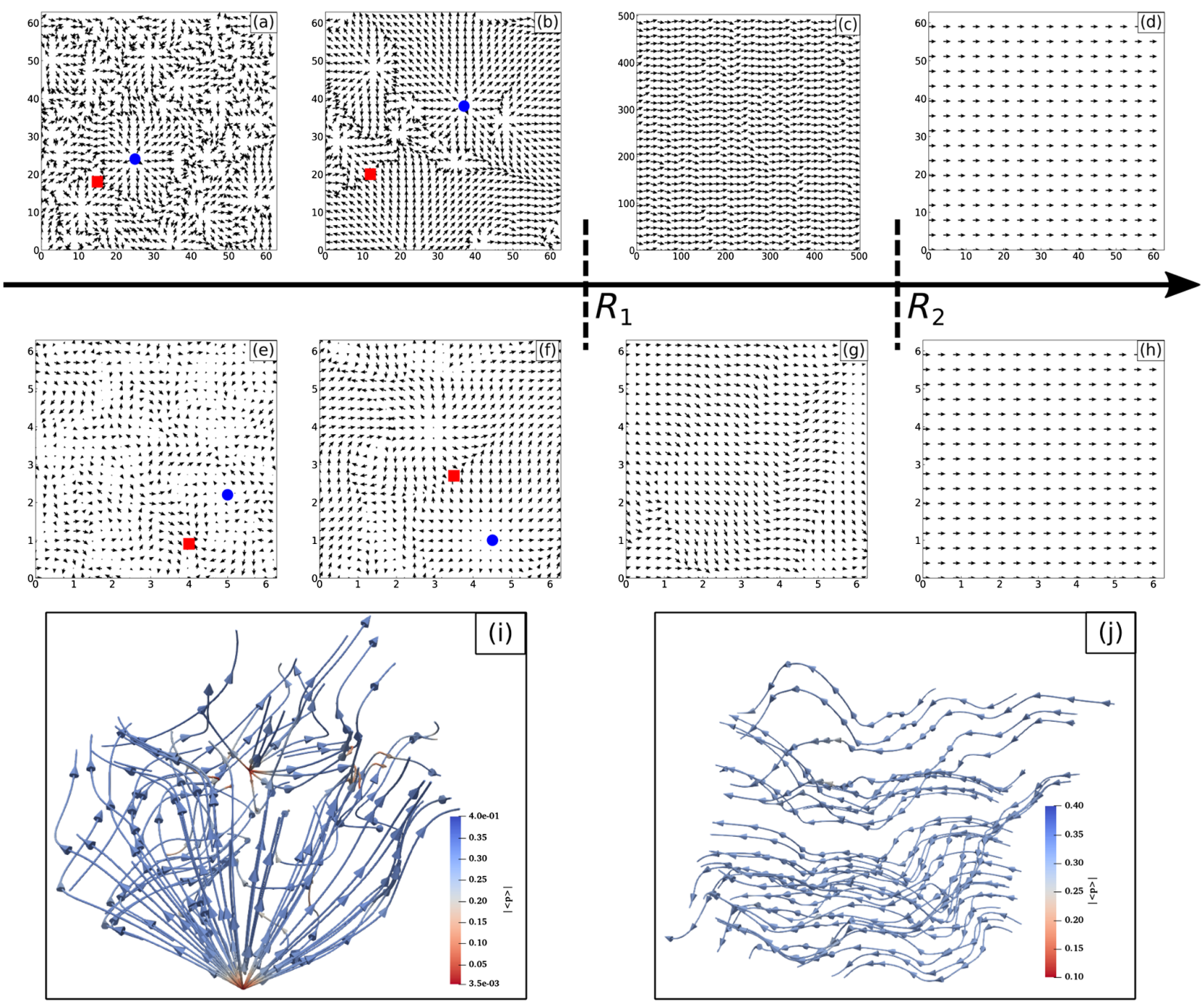

FIG. 4. Increasing interdefect distance as a function of $R$. Order parameter streamlines for 2D (runs SPP4, SPP6, and SPP9): (a) $R=0.1$, (b) $R=0.25$, (c) $R=8$, and (d) $R>12$; streamlines in $y=0$ plane for 3D (runs SPP1 and SPP3): (e) $R=0.02$, (f) $R=0.0625$, (g) $R=1.25$, and (h) $R>12$. Typical hedgehogs are marked with filled black circles, and red squares indicate saddles. (i) Enlarged view of the three-dimensional order parameter streamlines showing the complex patterns between a hedgehog-saddlehedgehog configuration in (f). (j) Three-dimensional nearly ordered configuration in the phase-turbulent regime in (g). 

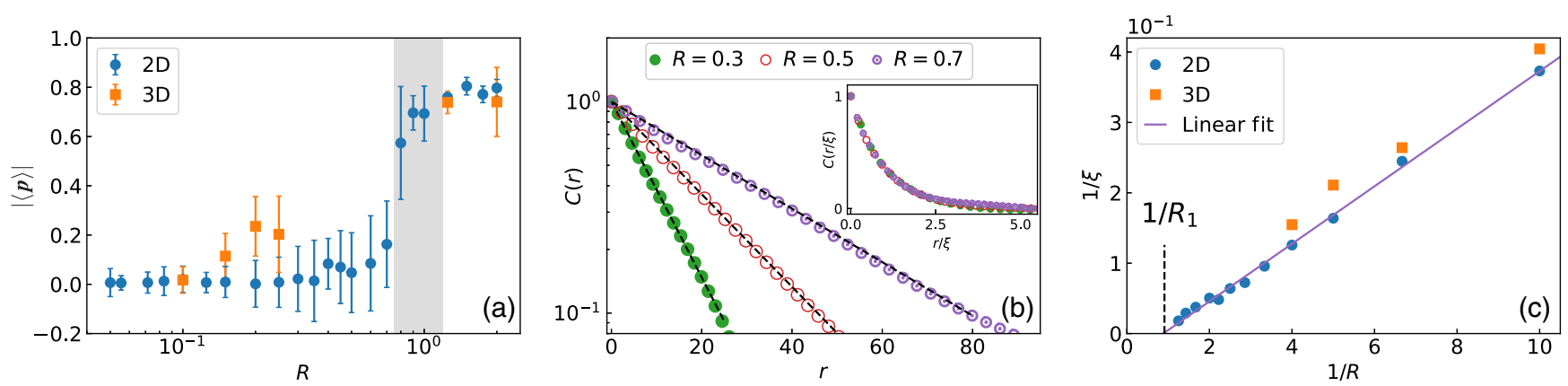

FIG. 5. (a) Variation of the order parameter $|\langle\boldsymbol{p}\rangle|$ with $R$ for our 2D and 3D simulations (see Table I), with the shaded region indicating the transition regime around $R=R_{1}$ as predicted by the linear stability analysis. For each data point, the spatiotemporal average is calculated from about 60 statistically independent realizations, and the standard deviation about the average is shown as the error bar. (b) Semilog plot of the correlation function $C(r)$ versus $r$ for $R=0.3,0.5$, and $0.7\left(R<R_{1}\right.$, runs SPP6-8). Dashed black lines indicate the exponential fit. Inset: collapse of steady-state correlation function when distance is scaled with the correlation length. (c) Plot of inverse correlation length $1 / \xi$ versus $1 / R$. The continuous purple line shows the linear fit to $2 \mathrm{D}$ data. Note that from the intercept of the linear fit on the horizontal axis we conclude that the correlation length diverges around $R=R_{1}$.

dependence of the positive Lyapunov spectrum to establish spatiotemporal chaos quantitatively. We do not know the mechanism that serves to preserve macroscopic flocking order despite the $O\left(q^{2}\right)$ instability. It appears that the growing amplitude of perturbations at small wave number $q$ triggers nonlinear effects which couple to large $q$ where the dynamics is stable. The behavior is reminiscent of that reported by Jayaprakash, Hayot, and Pandit [87] for the Kuramoto-Sivashinsky (KS) equation. The KS equation is a deterministic partial differential equation (PDE) with a negative diffusivity and, hence, a linear instability with growth rate $\propto q^{2}$ at small wave number $q$, peaking at a wave number $q_{*}$, stable behavior at large $q$ thanks to terms at higher order in $q$, and a nonlinearity that transfers weight from small to large $q$. Jayaprakash, Hayot, and Pandit [87] carry out a numerical coarse-graining, i.e., a spatial lowpass filtering, on the two-dimensional KS equation to show that the effective equations of motion for the modes with $q<q_{*}$ are those of a stochastic PDE with a positive diffusivity. It is possible that such a mechanism is at work in our case, but to settle this issue will require a treatment analogous to that of Ref. [87] for our substantially more complicated equations.

We now focus on the properties of the nonequilibrium phase transition. In Fig. 5(a), we plot the magnitude $|\langle\boldsymbol{p}\rangle|$ of the polar order parameter in the statistically steady state with increasing $R$, where angle brackets $\langle\cdot\rangle$ denote spatiotemporal averaging. For $R<R_{1},|\langle\boldsymbol{p}\rangle|$ is consistent with zero. We observe an onset of polar order once $R$ increases beyond $R_{1} \equiv 1+\lambda$. Figure 5(a) shows the order parameter for the largest system sizes studied; at large $R$, the values at half that system size are very similar. However, a detailed finite-size scaling analysis needs to be undertaken to find the correct scaling near the critical region [88].

In the defect-turbulence regime, we study the steadystate correlation function $C(r)=\langle\boldsymbol{p}(\boldsymbol{x}+\boldsymbol{r}) \cdot \boldsymbol{p}(\boldsymbol{x})\rangle /\left\langle\boldsymbol{p}(0)^{2}\right\rangle$, where the angular brackets denote spatial averaging. We plot the correlation function $C(r)$ versus $r$ in Fig. 5(b) and evaluate the correlation length by fitting an exponential decay $\exp (-r / \xi)$ to the numerical data [89]. We see that the correlation functions for different values of $R<R_{1}$ fall on a single curve plotted against $r / \xi$. Moreover, from Fig. 5(c), $\xi$ grows and possibly diverges as $R \rightarrow R_{1}$; our limited data points are consistent with an exponent of unity. Further progress requires finite-size scaling studies and measurements of order-parameter correlations at asymptotically small wave numbers [88] for $R>R_{1}$ to test the nature of the ordered state.

\section{Energy spectrum}

A state of complex, correlated but disorderly flow is seen in a wide variety of suspensions of motile organisms and motorized biofilaments. It is termed active turbulence and analyzed through the study of energy spectra as in conventional turbulence [16-18,26,29,32,34,36,90-93]. However, these studies all consider systems with negligible inertia. Here, we examine numerically the spatial power spectral densities for the polar order parameter and the hydrodynamic velocity field, in the defect- and phase-turbulent regimes-the latter owing its existence to inertia. In keeping with typical turbulence studies, we use the shell-averaged energy spectra of the velocity and the order parameter

$$
\begin{aligned}
& E_{u}(q)=\sum_{q-1 / 2 \leq|\boldsymbol{m}|<q+1 / 2}\left|\boldsymbol{u}_{\boldsymbol{m}}\right|^{2} \text { and } \\
& E_{p}(q)=\sum_{q-1 / 2 \leq|\boldsymbol{m}|<q+1 / 2}\left|\boldsymbol{p}_{\boldsymbol{m}}\right|^{2}
\end{aligned}
$$

where $\boldsymbol{u}_{\boldsymbol{m}}$ and $\boldsymbol{p}_{\boldsymbol{m}}$ are the Fourier coefficients of the velocity $\boldsymbol{u}$ and order parameter $\boldsymbol{p}$ fields.

Among the features of interest are Porod's law regimes corresponding to the fields of topological defects. In addition, for $R<R_{1}$, we find velocity correlations of 

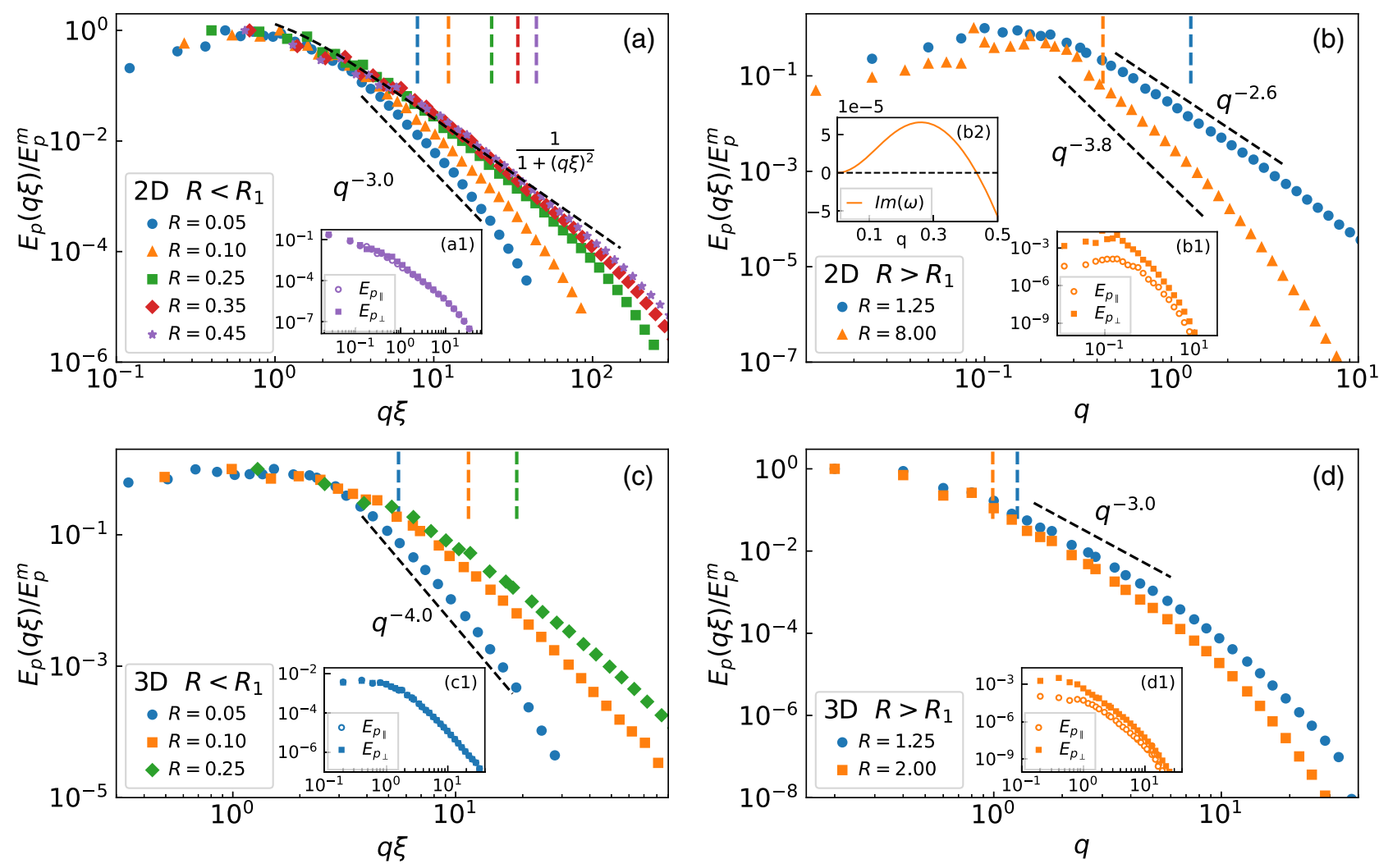

FIG. 6. Order parameter energy spectrum $E_{p}(q)$ for different values of $R$ for (a),(b) two-dimensional [runs SPP4, SPP6, SPP9, and SPP10] and (c),(d) three-dimensional [run SPP3] active suspension. For $R<R_{1}$ and $q \xi>1$, we observe a Porod's tail due to defects, i.e., $E_{p}(q) \sim q^{-3}$ in $2 \mathrm{D}$ and $E_{p}(q) \sim q^{-4}$ in 3D. As $R$ approaches $R_{1}$, we find $E_{p}(q) \sim 1 /\left[1+(q \xi)^{2}\right]$ consistent with the exponential decay of the correlation function. For $R_{1}<R<R_{2}, E_{p}(q) \sim q^{-2.6}$ for $R=1.25$, and the slope increases to $q^{-3.8}$ for $R=8$. For different values of $R$, dashed vertical lines (with the same color as markers) indicate the largest $q$ which is linearly unstable. Insets (a1), (b1), (c1), and (d1) show the spectra $E_{p_{\|}}$for components of $\boldsymbol{p}$ along the mean ordering direction and $E_{p_{\perp}}$ for a representative direction orthogonal to it. For $R<R_{1}$, fluctuations are isotropic, whereas for $R>R_{1}$, transverse fluctuations dominate, with $E_{p_{\perp}} \approx 10^{2} E_{p_{\|}}$. Note that, although the mean order parameter is, of course, consistent with zero for $R<R_{1}$, we use the numerically measured mean ordering direction to define $\|$ and $\perp$. Inset (b2) shows the growth rate for $R=8$. Note that only a small number of modes between $q=0$ and $q=0.5$ are linearly unstable.

Ornstein-Zernike form, with a correlation length much larger than that of the order parameter, whose origin we discuss below. For $R>R_{1}$, the phase-turbulent but ordered state, we present preliminary evidence of fluctuations of the broken-symmetry or Nambu-Goldstone mode. The behaviors of $E_{p}(q)$ and $E_{u}(q)$ for a range of values of $R$ are displayed in Figs. 6 and 7.

\section{Energy spectra of the order parameter}

We observe that, for $R<R_{1}$, the spectrum peaks around $q \xi \sim 1$. At moderate values of $R, 0.1<R<1$, because of exponential decay in orientational correlations, we expect $E_{p}(q) \sim 1 /\left(1+\xi^{2} q^{2}\right)$ for $q \xi>1$. On the other hand, for $R \ll 1$, because of defects, we expect a Porod's scaling [94] $E_{p}(q)=q^{-3}$ in two dimensions and $E_{p}(q)=q^{-4}$ in three dimensions for $q \xi \gg 1$. Recent studies on dry active matter [75] using a scale-by-scale budget analysis reveal that, even in the presence of defects, the nonlinear transfer mechanisms could lead to a non-Porod scaling. Unfortunately, we do not have sufficient scaling range-due to modest grid resolution at $R \ll R_{1}$ - to undertake such analysis. We emphasize that the quoted exponent values are empirically determined by conservatively selecting an appropriate dynamic range of wave numbers away from the smallest (approximately $1 / L$ ) and the largest, viz., $q_{K} \equiv 2 \pi / \ell_{K}$, beyond which Frank elasticity dominates.

In the phase-turbulent regime $R_{1}<R<R_{2}$, we observe $E_{p}(q) \sim q^{-3}$ for $R$ close to $R_{1}$. As $R$ approaches $R_{2}$, the range of linearly unstable modes shrinks and is restricted to wave numbers close to large scales [small $q$; see Fig. 6(b)]. For the linearly unstable modes, we observe a weak $q$ dependence, whereas for wave numbers outside the linearly unstable regime, the nonlinearities lead to a transfer of order-parameter fluctuations to small scales with a powerlaw spectrum $E_{p}(q) \sim q^{-3.8}$ [see Figs. 6(b) and 6(d)]. 

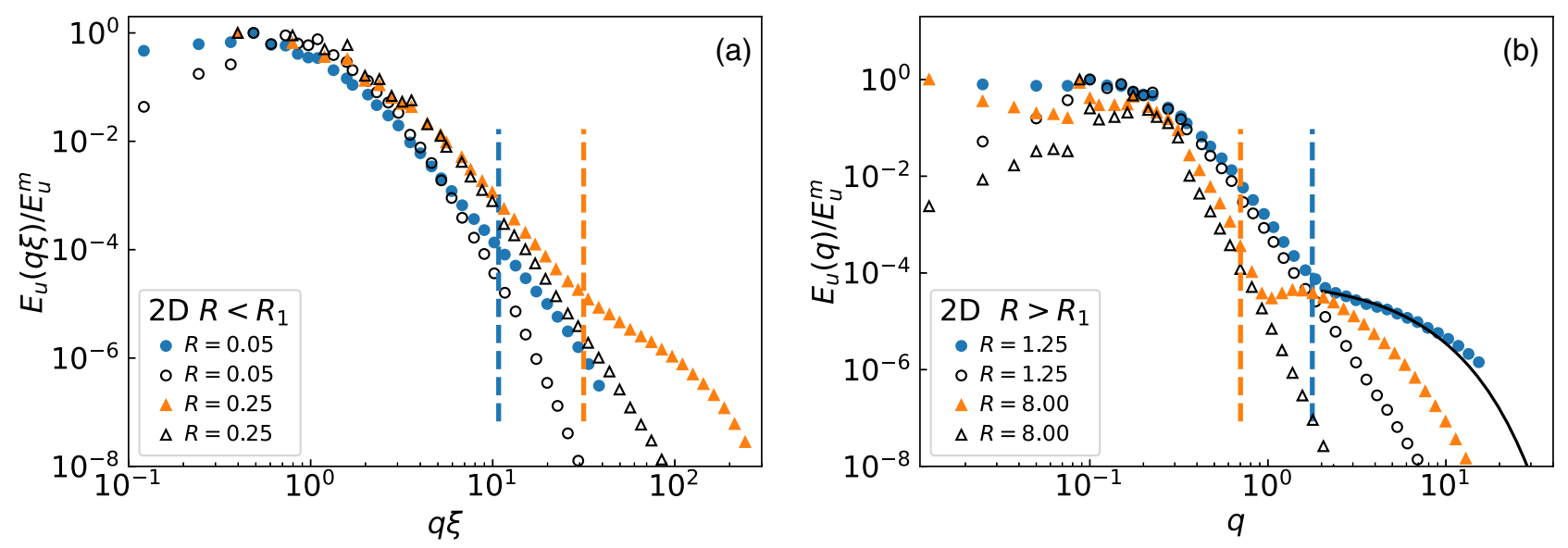

FIG. 7. Kinetic energy spectrum $E_{u}(q)$ (filled symbols) for different values of $R$ for 2D active suspension [runs SPP4, SPP9, and SPP10] for (a) $R<R_{1}$ and (b) $R>R_{1}$. Similar to the order parameter spectrum, we observe power-law behavior for $1<q \xi<q_{\sigma} \xi$, where $q_{\sigma} \equiv 2 \pi / \ell_{\sigma}$. For small $q$, we find a good agreement between the energy spectrum and the prediction $E_{u}(q) \sim\left[E_{p}(q)\right]^{2}($ unfilled symbols). For $R>R_{1}$ and large $q\left(q>q_{\sigma}\right)$, the energy spectrum shows an exponential decay $E_{u}(q) \sim \exp (-0.31 q)$ (black line). For different values of $R$, dashed vertical lines (with the same color as the markers) are drawn at $q=q_{\sigma}$.

\section{Energy spectra of the velocity}

In Eq. (1), we expect the dominant balance to be between acceleration and activity, as the Reynolds number obtained by comparing the advective and viscous terms, based on the root-mean-square hydrodynamic velocity, is small $\left(\operatorname{Re} \equiv \rho u_{\mathrm{rms}} \xi / \mu \leq 0.5\right)$ [95]. We, therefore, expect, for small $q, \omega u_{q} \sim \sigma_{a} q \sum_{k} \boldsymbol{p}_{k} \boldsymbol{p}_{q-k}$. If we assume that the dominant contribution to the convolution comes from terms with $|\boldsymbol{k}|=|\boldsymbol{q}-\boldsymbol{k}|=q$, i.e., on the same shell in Fourier space, we get $\omega u_{q} \sim \sigma_{a} q E_{p}(q)$. Using $\omega \sim v_{0} q$ [see Eq. (10)], we get $E_{u}(q) \sim\left(\sigma_{a} / v_{0}\right)^{2} E_{p}(q)^{2}$. The plot in Fig. 7(a) shows good agreement between $E_{u}(q)$ obtained from our DNS and the conjecture above for small $q$. For large $q>2 \pi / \ell_{\sigma}$, we expect viscous dissipation to be dominant, and, therefore, similar to the dissipation range in hydrodynamic turbulence, we expect an exponential decay in the energy spectrum $E_{u}(q) \sim \exp \left(-a k^{\delta}\right)[96,97]$. From our numerical simulations, we find $\delta=1$.

It is worth noting that, although turbulence in an apolar active suspension is controlled by half-integer defects in 2D $[17,18]$ and disclination loops or line defects in 3D [98,99], the flow energy spectrum $E_{u}(q) \sim q^{-3.5}-q^{-5}$ reported in those works on active nematics does not differ drastically from our observation $E_{u}(q) \sim q^{-4.8}-q^{-6}$ for defect turbulence in the $R<R_{1}$ regime in our polar system.

\section{Energy spectra for $R \ll R_{1}$}

For $R \ll R_{1}$, the interdefect separation $\xi$ is comparable to $\ell_{\sigma}$; however, for length scales much larger than $\xi$, the system should in effect be an unsteady Stokes fluid with fluctuating stresses with short-ranged spatial correlation and with a correlation time $\tau$. In such a scenario, it is straightforward to show $[100,101]$ that the equal-time velocity correlator has Ornstein-Zernike form, so that $E_{u}(q) \sim q^{D-1} /\left[\left(q \ell_{\tau}\right)^{2}+1\right]$ for $q \xi \ll 1$, where $\ell_{\tau}=\sqrt{\mu \tau / \rho}$ is the distance vorticity diffuses in a time $\tau$. Note: (a) although the analysis of Ref. [100] contains this result, they do not emphasize the distinct roles of $\xi$ and $\ell_{\tau}$. (b) The power-law correlations discussed in the inertialess treatment of Refs. [93,102] amount to the $q \ell_{\tau} \gg 1$ regime of the above.

To investigate this regime, we perform high-resolution simulations in two dimensions with large system size and small $R=0.01$ (run SPP11) to ensure $\mathcal{L} \gg \xi$. The plot in Fig. 8(a) shows that $E_{p}(q) \sim q$ for $q \xi \ll 1$, indicating that order parameter fluctuations are uncorrelated. We, therefore, expect the active stresses to be spatially uncorrelated. Consistent with the arguments above, the plot in Fig. 8(b) shows that the kinetic energy spectrum follows $E_{u}(q) \sim$ $q /\left[\left(q \ell_{\tau}\right)^{c}+1\right]$ with $c \approx 2.3$ (obtained from a least-squares fit) close to the theoretically predicted value $c=2$. Note that the length scale $\ell_{\tau}$ is larger than the interdefect separation, $\ell_{\tau} / \xi \approx 3$. As $R \rightarrow 0$, we expect $\xi \rightarrow 0$ and $\ell_{\tau} \gg \xi$; therefore, the peak of spectra in Fig. 8(b) would shift to very small $q \sim 1 / \ell_{\tau}$. Thus, our analysis naturally recovers and explains the recently observed $E_{u}(q) \sim q^{-1}$ scaling of active nematic turbulence in the Stokesian regime [93].

\section{Evidence for the broken-symmetry mode?}

We close our discussion of energy spectra with a speculation backed by qualitative numerical measurements. In any noisy ordered state in which a continuous symmetry has been spontaneously broken, the spatial power spectral density should contain information about the brokensymmetry modes, i.e., the components of the order parameter field perpendicular to the mean ordering direction, whose variance should diverge at a small wave number $[56,103,104]$. This variance should be seen in the energy spectrum in the ordered but noisy phase-turbulent state we 



FIG. 8. Energy spectra for $R=0.01$. (a) Order parameter energy spectra $E_{p}(q \xi)$. The black dashed line indicates $E_{p}(q) \sim q$ scaling. (b) Kinetic energy spectra $E_{u}(q \xi)$. A least-squares fit to the curve $a q /\left[\left(q \ell_{\tau}\right)^{c}+1\right]$ gives $a=1.6 \times 10^{-5}, c=2.3$, and $\ell_{\tau}=1$.

observe for $R_{1}<R<R_{2}$. We offer preliminary evidence for such fluctuations. Insets (b1) and (d1) in Fig. 6 show that for $R>R_{1}$ the contributions to the energy spectrum from components of $\boldsymbol{p}$ in a representative $(\perp)$ direction perpendicular to the mean direction of ordering far outweigh those from components in the ordering direction, especially at small $q$. This result is consistent with the expectations of an enhanced variance mentioned above. We check for consistency that the spectra for the disordered phase for $R<R_{1}$ [insets (a1) and (c1) in Fig. 6] show no such anisotropy.

A quantitative study of the spectrum of fluctuations at small wave number, to test whether the regime $R_{1}<R<$ $R_{2}$ has the classic features of a broken-symmetry phase, makes high computational demands. The wavelength at which the linear instability growth rate is maximum can be viewed as the scale of energy injection and, therefore, as the small-scale cutoff for a long-wavelength study of the ordered phase. At the same time, scales substantially smaller than this cutoff must be resolved so that the instability and the nonlinear effects leading to phase turbulence can operate. If such a simulation is realized, the approach of choice would be to emulate [87] and construct an effective stochastically forced theory for the small- $q$ modes via numerical coarse-graining.

\section{SUMMARY AND PROSPECT}

We have shown that extensile active polar liquid crystals and swimmer suspensions can outswim their viscous instability. Their fate is governed by a control parameter $R$, the ratio of the inertia of self-advection to the scale of active stress. Our stability analysis and numerical studies find evidence for a continuous flocking transition with a growing correlation length as $R$ increases past a threshold of the order of unity, from hedgehog-defect turbulence to a noisy but ordered phase-turbulent state. A quiescent, linearly stable ordered state sets in at larger $R$. These dramatic advances in the theory of flocks in fluid, whose instability [10] can now be seen as simply the Stokesian limit of a rich phase diagram, should stimulate a new wave of experiments on swimmers at nonzero Reynolds number. Important directions for the near future are studies of finitesize scaling and long-wavelength order-parameter correlations for $R>R_{1}$ to establish the nature of the ordered phase; the construction of an effective stochastic theory for the long-wavelength modes, as carried out [87] for the Kuramoto-Sivashinsky $[105,106]$ equation; the inclusion of active-particle concentration; the contribution of other polar terms to the dynamics; and the effect of added random forcing on our phase diagram. Meanwhile, we look forward to tests of our theory in experiments on collections of swimmers at small but nonzero Reynolds number [45], as well as particle-based numerical simulations featuring, for example, collections of "spherobots" [107,108].

\section{ACKNOWLEDGMENTS}

S. R. was supported by a J. C. Bose Fellowship of the SERB (India) and by the Tata Education and Development Trust. R. C. acknowledges the support of the SERB (India). R. C., N.R., and P. P. acknowledge support from the Department of Atomic Energy (DAE), Government of India, under Project Identification No. RTI 4007. The simulations were performed using resources provided by TIFR, Hyderabad. We thank $\mathrm{H}$ Chaté for valuable comments on an early draft. R.C., N.R., P.P., and S.R. acknowledge the contributions of Aditi Simha, now sadly deceased, to the development and completion of this work.

\section{APPENDIX A: STABILITY ANALYSIS WITH CONCENTRATION}

We now present the linear stability analysis in presence of the concentration field. Linearizing Eqs. (1)-(3) about the base state $(\boldsymbol{u}=0, \boldsymbol{p}=\hat{\boldsymbol{x}}, c=1)$, we get 

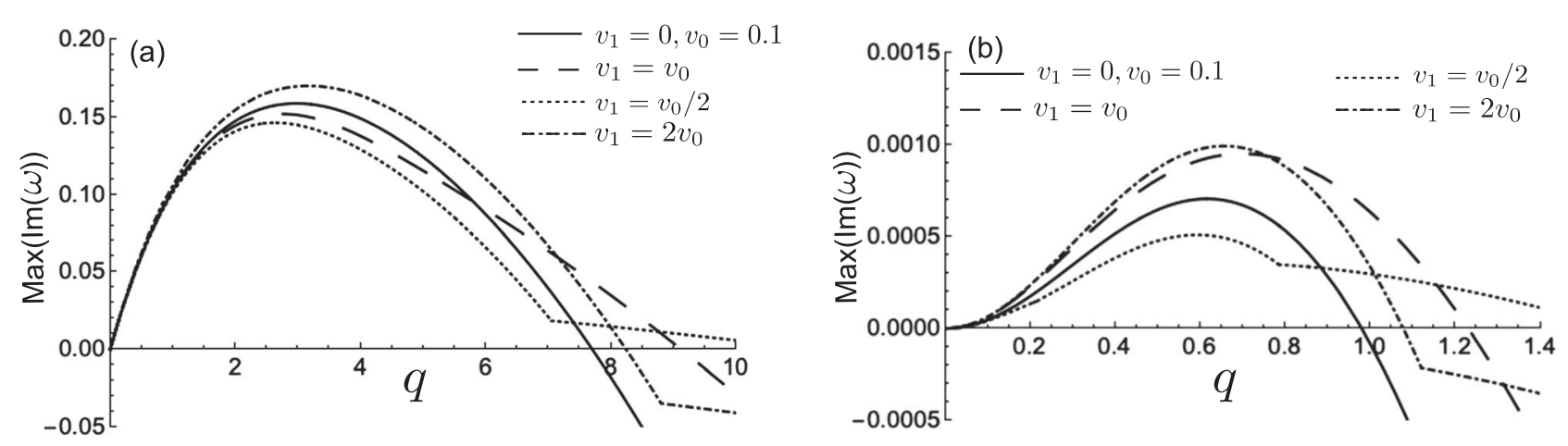

FIG. 9. Maximum growth rate of the unstable splay-bend modes for different values of $v_{0}$ and $v_{1}$ for (a) $O(q): R=0.1$ and (b) $O\left(q^{2}\right): R=5$ regimes. Note that the dispersion relation for $v_{1}=0$ is identical to Eq. (10) (see the discussion below). The other parameters are $\phi=15^{\circ}, \mu=10^{-1}, \Gamma K=10^{-3}, \lambda=0.1$, and $E=0.05$.

$$
\begin{aligned}
\left(\rho \partial_{t}+\mu q^{2}\right) \delta \boldsymbol{u}_{\perp \boldsymbol{q}}= & -i \mathbf{T}_{\boldsymbol{q}} \cdot\left[\left(\sigma_{a}+\frac{\lambda-1}{2} K q^{2}\right) \hat{\boldsymbol{x}} \boldsymbol{q}_{\perp}\right. \\
& \left.+q_{x}\left(\sigma_{a}+\frac{\lambda+1}{2} K q^{2}\right) \mathbf{I}\right] \cdot \delta \boldsymbol{p}_{\perp \boldsymbol{q}} \\
& -i \mathbf{T}_{\boldsymbol{q}} \cdot\left(\sigma_{a} q_{x} \hat{\boldsymbol{x}}\right) \delta c_{\boldsymbol{q}} \\
\partial_{t} \delta \boldsymbol{p}_{\perp \boldsymbol{q}}= & +i\left(\frac{\lambda+1}{2} q_{x} \mathbf{I}-\frac{\lambda-1}{2} \frac{\boldsymbol{q}_{\perp} \boldsymbol{q}_{\perp}}{q_{x}}\right) \cdot \delta \boldsymbol{u}_{\perp \boldsymbol{q}} \\
& -\left(i v_{0} q_{x}+\Gamma K q^{2}\right) \delta \boldsymbol{p}_{\perp \boldsymbol{q}}+i \boldsymbol{q}_{\perp} E \delta c_{\boldsymbol{q}}, \\
& \left(\partial_{t}+i v_{1} q_{x}\right) \delta c_{\boldsymbol{q}}=-i v_{1} \boldsymbol{q}_{\perp} \cdot \boldsymbol{\delta} \boldsymbol{p}_{\perp \boldsymbol{q}} .
\end{aligned}
$$

The dispersion relations for Eqs. (A1)-(A3) are obtained using the same procedure as highlighted in Sec. II B. The growth rate of the twist-bend modes is the same as Eq. (11), because the terms containing concentration fluctuations in Eqs. (A1) and (A2) point in the direction of $\boldsymbol{q}_{\perp}$. The growth rate of the splay-bend modes is identical to Eq. (10) for $v_{1}=0$, because concentration fluctuations decouple from the orientation and velocity distortions. For $v_{1} \neq 0$, the splay-bend modes couple to the concentration fluctuations and are obtained by taking in-plane divergence $\left(\nabla_{\perp} \cdot\right)$ on Eqs. (A1) and (A2). We compare the most unstable growth rate in Fig. 9 and show that the $O(q)$ and the $O\left(q^{2}\right)$ behavior at remains unaltered small $q$.

\section{APPENDIX B: STABILITY ANALYSIS INCLUDING THE NEGLECTED POLAR TERMS}

We now explain the role of the additional polar terms on the stability thresholds. We first recall the complete Eqs. (1) and II A with the polar terms:

$$
\begin{aligned}
\rho\left(\partial_{t} \boldsymbol{u}+\boldsymbol{u} \cdot \nabla \boldsymbol{u}\right) & =-\nabla P+\mu \nabla^{2} \boldsymbol{u}+\nabla \cdot\left(\boldsymbol{\Sigma}^{a}+\boldsymbol{\Sigma}^{r}\right) \text { and } \\
\partial_{t} \boldsymbol{p}+\left(\boldsymbol{u}+v_{0} \boldsymbol{p}\right) \cdot \nabla \boldsymbol{p} & =\lambda \boldsymbol{S} \cdot \boldsymbol{p}+\boldsymbol{\Omega} \cdot \boldsymbol{p}+\Gamma \boldsymbol{h}+\ell \nabla^{2} \boldsymbol{u},
\end{aligned}
$$

where $\boldsymbol{\Sigma}^{a} \equiv-\sigma_{a} \boldsymbol{p} \boldsymbol{p}-\gamma_{a}\left(\nabla \boldsymbol{p}+\nabla \boldsymbol{p}^{T}\right), \boldsymbol{\Sigma}^{r} \equiv[(1-\lambda) \boldsymbol{p h}-$ $(\lambda+1) \boldsymbol{h} \boldsymbol{p}] / 2-\ell\left(\nabla \boldsymbol{h}+\nabla \boldsymbol{h}^{T}\right)$, and $\boldsymbol{h}=-\delta F / \delta \boldsymbol{p}$. These are sufficient for our current purpose, because we already establish in Sec. II B 1 that for extensile systems, which is our main focus, the dominant failure modes are the twistbend modes which are always decoupled from concentration fluctuations. The dispersion relation for these modes with these terms is

$$
\begin{aligned}
\omega_{ \pm}^{t}= & \frac{1}{2} v_{0} q \cos \phi-i \frac{\mu_{+}}{2 \rho} q^{2} \pm\left(\frac{\sigma_{a}}{2 \rho}\right)^{1 / 2}\left[A(0) \cos ^{2} \phi q^{2}\right. \\
& +i B(0)\left(1-\frac{\gamma_{a}(1+\lambda)+2 \ell \sigma_{a}}{v_{0} \mu_{-}}\right) \cos \phi q^{3} \\
& \left.+\left(\bar{G}(\phi)+\frac{2 \gamma_{a} \ell}{\sigma_{a}}\right) q^{4}\right]^{1 / 2}
\end{aligned}
$$

where $R \equiv \rho v_{0}^{2} / 2 \sigma_{a}, \mu_{ \pm}=\mu(1 \pm \beta), \beta=\Gamma K \rho / \mu, B(\phi)=$ $\left(v_{0} \mu_{-} / \sigma_{a}\right) \cos \phi, \quad G(\phi)=-\left(\mu_{-}^{2} / 2 \rho \sigma_{a}\right)+\left(K / 2 \sigma_{a}\right)(1+$ $\lambda \cos 2 \phi)^{2}$, and $\bar{G}(\phi)=-\left(\mu_{-}^{2} / 2 \rho \sigma_{a}\right)+\left(K / 2 \sigma_{a}\right)(1+$ $\lambda)^{2} \cos ^{2} \phi$. Comparing Eq. (B1) to the dispersion relation for $\gamma_{a}, \ell=0$ [Eq. (11) of the main text]

$$
\begin{aligned}
\omega_{ \pm}^{t}= & \frac{1}{2} v_{0} q \cos \phi-i \frac{\mu_{+}}{2 \rho} q^{2} \pm\left(\frac{\sigma_{a}}{2 \rho}\right)^{1 / 2} \\
& \times\left[A(0) \cos ^{2} \phi q^{2}+i B(0) \cos \phi q^{3}+\bar{G}(\phi) q^{4}\right]^{1 / 2},
\end{aligned}
$$

and working to order $q^{2}$ we see that the transition between $O(q)$ and $O\left(q^{2}\right)$ instabilities, which is determined by $A(0)$, remains unchanged and that the relative shifts of the coefficients of $q^{2}$ in the mode frequencies are of the order of

$$
D_{1}=\gamma_{a} / \mu v_{0}, \quad D_{2}=\ell \sigma_{a} / \mu v_{0},
$$

which resemble inverse capillary numbers given that $\gamma_{a}$ and $\ell \sigma_{a}$ have units of surface tension. The length $\ell$ controls 


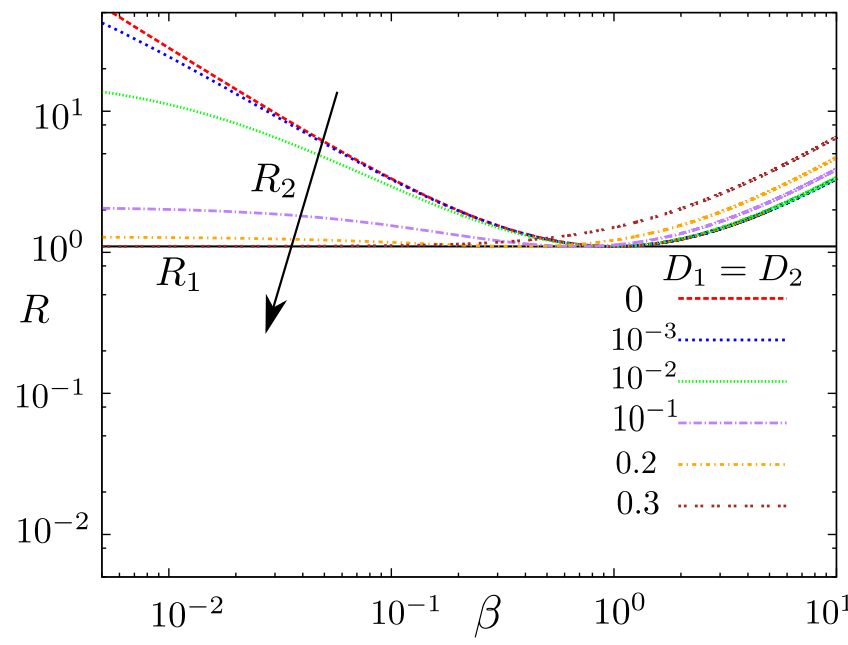

FIG. 10. $R-\beta$ stability diagram including polar contributions $\ell$ and $\gamma_{a}$ to flow alignment and active stress. The line at $R=R_{1}$ denoting the junction between the $O(q)$ and the $O\left(q^{2}\right)$ instabilities remains unchanged. However, the line $R=R_{2}$, which marks the borderline between the $O\left(q^{2}\right)$ instability and the stable aligned state, starts to deviate as the active capillary numbers decrease from very large values toward values of the order of unity.

polar couplings of orientation to flow in the passive theory and, as such, should be related, in a microscopic theory, to a geometrical measure of polarity of the constituent particles, such as a fore-aft size difference. $\gamma_{a}$, with units of force per unit length, governs polar active contributions to the stress tensor. It is then reasonable to expect that $\gamma_{a} \sim \sigma_{a} \ell$ and, thus, that $D_{1}$ and $D_{2}$ are similar in magnitude. For particles of size $b$, the viscosity-based estimate $\sigma_{a} \sim \mu v_{0} / b$ implies $D_{1}, D_{2} \sim \ell / b$, while an inertia-based estimate $\sigma_{a} \sim \rho v_{0}^{2}$ yields $D_{1}, D_{2} \sim \ell \operatorname{Re} / b$, where $\operatorname{Re}=\rho v_{0} b / \mu$ is the Reynolds number at the particle scale. With either estimate, $D_{1}$ and $D_{2}$ should typically be small, because $\ell / b$ is the ratio of a fore-aft size difference to an overall size. Figure 10 shows the results of linear stability analysis for nonzero $D_{1}=D_{2}$. The $O(q)$-unstable, $O\left(q^{2}\right)$-unstable, and linearly stable regimes exist over the entire range; for $D_{1}, D_{2} \ll 1$ the linear stability analysis differs negligibly from that with $\gamma_{a}, \ell=0$; and the $O\left(q^{2}\right)$-unstable regime shrinks in extent as $D_{1}$ and $D_{2}$ grow to values of the order of unity. We therefore expect the qualitative features of the dynamical phase diagram as seen in our numerical studies to persist for nonzero $\gamma_{a}$ and $\ell$, but we have not carried out the corresponding direct numerical solutions of the hydrodynamic equations.

[1] S. Ramaswamy, The Mechanics and Statistics of Active Matter, Annu. Rev. Condens. Matter Phys. 1, 323 (2010).

[2] M. C. Marchetti, J.F. Joanny, S. Ramaswamy, T. B. Liverpool, J. Prost, M. Rao, and R. A. Simha, Hydrodynamics of Soft Active Matter, Rev. Mod. Phys. 85, 1143 (2013).
[3] J. Toner, Y. Tu, and S. Ramaswamy, Hydrodynamics and Phases of Flocks, Ann. Phys. (Amsterdam) 318, 170 (2005), special issue.

[4] S. Ramaswamy, Active Fluids, Nat. Rev. Phys. 1, 640 (2019).

[5] J. Prost, F. Jülicher, and J. F. Joanny, Active Gel Physics, Nat. Phys. 11, 111 (2015).

[6] F. Jülicher, K. Kruse, J. Prost, and J. F. Joanny, Active Behavior of the Cytoskeleton, Phys. Rep. 449, 3 (2007).

[7] F. Jlicher, S. W. Grill, and G. Salbreux, Hydrodynamic Theory of Active Matter, Rep. Prog. Phys. 81, 076601 (2018).

[8] D. Marenduzzo, An Introduction to the Statistical Physics of Active Matter: Motility-Induced Phase Separation and the Generic Instability of Active Gels, Eur. Phys. J. Special Topics 225, 2065 (2016).

[9] G. Gompper et al., The 2020 Motile Active Matter Roadmap, J. Phys. Condens. Matter 32, 193001 (2020).

[10] R. Simha and S. Ramaswamy, Hydrodynamic Fluctuations and Instabilities in Ordered Suspensions of Self-Propelled Particles, Phys. Rev. Lett. 89, 058101 (2002).

[11] R. Voituriez, J. F. Joanny, and J. Prost, Spontaneous Flow Transition in Active Polar Gels, Europhys. Lett. 70, 404 (2005).

[12] B. Martínez-Prat, J. Ignés-Mullol, J. Casademunt, and F. Sagués, Selection Mechanism at the Onset of Active Turbulence, Nat. Phys. 15, 362 (2019).

[13] G. Duclos, C. Blanch-Mercader, V. Yashunsky, G. Salbreux, J. F. Joanny, J. Prost, and P. Silberzan, Spontaneous Shear Flow in Confined Cellular Nematics, Nat. Phys. 14, 728 (2018).

[14] D. Saintillan, Rheology of Active Fluids, Annu. Rev. Fluid Mech. 50, 563 (2018).

[15] L. Giomi, M. C. Marchetti, and T. B. Liverpool, Complex Spontaneous Flows and Concentration Banding in Active Polar Films, Phys. Rev. Lett. 101, 198101 (2008).

[16] L. Giomi and M. C. Marchetti, Polar Patterns in Active Fluids, Soft Matter 8, 129 (2012).

[17] L. Giomi, Geometry and Topology of Turbulence in Active Nematics, Phys. Rev. X 5, 031003 (2015).

[18] J. Urzay, A. Doostmohammadi, and J. Yeomans, Multiscale Statistics of Turbulence Motorized by Active Matter, J. Fluid Mech. 822, 762 (2017).

[19] E. Lauga and R.E. Goldstein, Dance of the Microswimmers, Phys. Today 65, No. 9, 30 (2012).

[20] K. Kruse, J. F. Joanny, F. Jülicher, J. Prost, and K. Sekimoto, Asters, Vortices, and Rotating Spirals in Active Gels of Polar Filaments, Phys. Rev. Lett. 92, 078101 (2004).

[21] L. Giomi, M. J. Bowick, X. Ma, and M. C. Marchetti, Defect Annihilation and Proliferation in Active Nematics, Phys. Rev. Lett. 110, 228101 (2013).

[22] J. Elgeti, M. E. Cates, and D. Marenduzzo, Defect Hydrodynamics in 2D Polar Active Fluids, Soft Matter 7, 3177 (2011).

[23] A. Doostmohammadi, T. N. Shendruk, K. Thijssen, and J. M. Yeomans, Onset of Meso-scale Turbulence in Active Nematics, Nat. Commun. 8, 15326 (2017).

[24] S. P. Thampi, R. Golestanian, and J. M. Yeomans, Vorticity, Defects and Correlations in Active Turbulence, Phil. Trans. R. Soc. A 372, 20130366 (2014). 
[25] L. Giomi, M. J. Bowick, P. Mishra, R. Sknepnek, and M. C. Marchetti, Defect Dynamics in Active Nematics, Phil. Trans. R. Soc. A 372, 20130365 (2014).

[26] T. Sanchez, D. T. N. Chen, S. Decamp, M. Heymann, and Z. Dogic, Spontaneous Motion in Hierarchically Assembled Active Matter, Nature (London) 491, 431 (2012).

[27] S. Decamp, G. Redner, A. Baskaran, M. Hagan, and Z. Dogic, Orientational Order of Motile Defects in Active Nematics, Nat. Mater. 14, 1110 (2015).

[28] D. Saintillan and M. J. Shelley, Orientational Order and Instabilities in Suspensions of Self-Locomoting Rods, Phys. Rev. Lett. 99, 058102 (2007).

[29] C. W. Wolgemuth, Collective Swimming and the Dynamics of Bacterial Turbulence, Biophys. J. 95, 1564 (2008).

[30] A. Doostmohammadi, J. Ignes-Mullol, J. Yeomans, and F. Sagus, Active Nematics, Nat. Commun. 9, 3246 (2018).

[31] C. Dombrowski, L. Cisneros, S. Chatkaew, R. E. Goldstein, and J.O. Kessler, Self-Concentration and Large-Scale Coherence in Bacterial Dynamics, Phys. Rev. Lett. 93, 098103 (2004).

[32] T. Ishikawa, N. Yoshida, H. Ueno, M. Wiedeman, Y. Imai, and T. Yamaguchi, Energy Transport in a Concentrated Suspension of Bacteria, Phys. Rev. Lett. 107, 028102 (2011).

[33] A. Sokolov and I. S. Aranson, Physical Properties of Collective Motion in Suspensions of Bacteria, Phys. Rev. Lett. 109, 248109 (2012).

[34] H. H. Wensink, J. Dunkel, S. Heidenreich, K. Drescher, R. E. Goldstein, H. Löwen, and J. M. Yeomans, Mesoscale Turbulence in Living Fluids, Proc. Natl. Acad. Sci. U.S.A. 109, 14308 (2012).

[35] J. Dunkel, S. Heidenreich, K. Drescher, H. H. Wensink, M. Bär, and R. E. Goldstein, Fluid Dynamics of Bacterial Turbulence, Phys. Rev. Lett. 110, 228102 (2013).

[36] V. Bratanov, F. Jenko, and E. Frey, New Class of Turbulence in Active Fluids, Proc. Natl. Acad. Sci. U.S.A. 112, 15048 (2015).

[37] D. J. Needleman and M. Shelley, The Stormy Fluid Dynamics of the Living Cell, Phys. Today 72, No. 9, 32 (2019).

[38] E. Tjhung, M. Cates, and D. Marenduzzo, Nonequilibrium Steady States in Polar Active Fluids, Soft Matter 7, 7453 (2011).

[39] B. L. Partridge, The Structure and Function of Fish Schools, Sci. Am. 246, 114 (1982).

[40] D. Weihs, Hydromechanics of Fish Schooling, Nature (London) 241, 290 (1973).

[41] A. D. Becker, H. Masoud, J. W. Newbolt, M. Shelley, and L. Ristroph, Hydrodynamic Schooling of Flapping Swimmers, Nat. Commun. 6, 8514 (2015).

[42] A. Filella, F. Nadal, C. Sire, E. Kanso, and C. Eloy, Model of Collective Fish Behavior with Hydrodynamic Interactions, Phys. Rev. Lett. 120, 198101 (2018).

[43] S. Wang and A. M. Ardekani, Unsteady Swimming of Small Organisms, J. Fluid Mech. 702, 286 (2012).

[44] G. Li, A. Ostace, and A. M. Ardekani, Hydrodynamic Interaction of Swimming Organisms in an Inertial Regime, Phys. Rev. E 94, 053104 (2016).
[45] D. Klotsa, As Above, So Below, and Also In Between: Mesoscale Active Matter in Fluids, Soft Matter 15, 8946 (2019).

[46] A. Attanasi, A. Cavagna, L. Del Castello, I. Giardina, T. S. Grigera, A. Jelić, S. Melillo, L. Parisi, O. Pohl, E. Shen et al., Information Transfer and Behavioural Inertia in Starling Flocks, Nat. Phys. 10, 691 (2014).

[47] A. Cavagna, I. Giardina, T. S. Grigera, A. Jelic, D. Levine, S. Ramaswamy, and M. Viale, Silent Flocks: Constraints on Signal Propagation across Biological Groups, Phys. Rev. Lett. 114, 218101 (2015).

[48] K. H. Nagai, Y. Sumino, R. Montagne, I. S. Aranson, and H. Chaté, Collective Motion of Self-Propelled Particles with Memory, Phys. Rev. Lett. 114, 168001 (2015).

[49] L. P. Dadhichi, J. Kethapelli, R. Chajwa, S. Ramaswamy, and A. Maitra, Nonmutual Torques and the Unimportance of Motility for Long-Range Order in Two-Dimensional Flocks, Phys. Rev. E 101, 052601 (2020).

[50] E. Lauga and T. R. Powers, The Hydrodynamics of Swimming Microorganisms, Rep. Prog. Phys. 72, 096601 (2009).

[51] H. Chaté and P. Manneville, in Turbulence (Springer, Boston, 1995), chapter on phase turbulence.

[52] B. Shraiman, A. Pumir, W. van Saarloos, P. Hohenberg, H. Chaté, and M. Holen, Spatiotemporal Chaos in the One-Dimensional Complex Ginzburg-Landau Equation, Physica (Amsterdam) 57D, 241 (1992).

[53] P. Manneville and H. Chaté, Phase Turbulence in the TwoDimensional Complex Ginzburg-Landau Equation, Physica (Amsterdam) 96D, 30 (1996).

[54] H. Chaté and P. Manneville, Phase Diagram of the TwoDimensional Complex Ginzburg-Landau Equation, Physica (Amsterdam) 224A, 348 (1996), dynamics of complex systems.

[55] L. P. Dadhichi, A. Maitra, and S. Ramaswamy, Origins and Diagnostics of the Nonequilibrium Character of Active Systems, J. Stat. Mech. (2018) 123201.

[56] P. C. Martin, O. Parodi, and P. S. Pershan, Unified Hydrodynamic Theory for Crystals, Liquid Crystals, and Normal Fluids, Phys. Rev. A 6, 2401 (1972).

[57] Y. Nambu and G. Jona-Lasinio, Dynamical Model of Elementary Particles Based on an Analogy with Superconductivity. I, Phys. Rev. 122, 345 (1961).

[58] J. Goldstone, Field Theories with 《SSuperconductor》》 Solutions, Nuovo Cimento 19, 154 (1961).

[59] X. Chen, E. Korblova, D. Dong, X. Wei, R. Shao, L. Radzihovsky, M. A. Glaser, J. E. Maclennan, D. Bedrov, D. M. Walba, and N. A. Clark, First-Principles Experimental Demonstration of Ferroelectricity in a Thermotropic Nematic Liquid Crystal: Polar Domains and Striking Electro-Optics, Proc. Natl. Acad. Sci. U.S.A. 117, 14021 (2020).

[60] A. Maitra, P. Srivastava, M. Rao, and S. Ramaswamy, Activating Membranes, Phys. Rev. Lett. 112, 258101 (2014) (Supplemental Material, Sec. II).

[61] A. Maitra, P. Srivastava, M. C. Marchetti, S. Ramaswamy, and M. Lenz, Swimmer Suspensions on Substrates: Anomalous Stability and Long-Range Order, Phys. Rev. Lett. 124, 028002 (2020). 
[62] M. C. Marchetti and T. N. Liverpool, in Cell Motility, edited by P. Lenz (Springer-Verlag, Berlin, 2007).

[63] W. Kung, M. C. Marchetti, and K. Saunders, Hydrodynamics of Polar Liquid Crystals, Phys. Rev. E 73, 031708 (2006).

[64] E. Tjhung, D. Marenduzzo, and M. E. Cates, Spontaneous Symmetry Breaking in Active Droplets Provides a Generic Route to Motility, Proc. Natl. Acad. Sci. U.S.A. 109, 12381 (2012).

[65] J. Toner and Y. Tu, Flocks, Herds, and Schools: A Quantitative Theory of Flocking, Phys. Rev. E 58, 4828 (1998).

[66] P. de Gennes and J. Prost, The Physics of Liquid Crystals, International Series of Monographs on Physics (Clarendon, Oxford, 1993).

[67] Y. Hatwalne, S. Ramaswamy, M. Rao, and R. A. Simha, Rheology of Active-Particle Suspensions, Phys. Rev. Lett. 92, 118101 (2004).

[68] K. Kruse, J. F. Joanny, F. Jülicher, J. Prost, and K. Sekimoto, Generic Theory of Active Polar Gels: A Paradigm for Cytoskeletal Dynamics, Eur. Phys. J. E 16, 5 (2005).

[69] We assume that concentrations are high enough that $F$ globally favors a nonzero magnitude for $p$. We, therefore, ignore $c$ dependence in the polynomial part of $F$.

[70] F. C. Frank, I. Liquid Crystals. On the Theory of Liquid Crystals, Discuss. Faraday Soc. 25, 19 (1958).

[71] H. Zocher, The Effect of a Magnetic Field on the Nematic State, Trans. Faraday Soc. 29, 945 (1933).

[72] C. W. Oseen, The Theory of Liquid Crystals, Trans. Faraday Soc. 29, 883 (1933).

[73] D. Forster, Microscopic Theory of Flow Alignment in Nematic Liquid Crystals, Phys. Rev. Lett. 32, 1161 (1974).

[74] H. Stark and T. C. Lubensky, Poisson-Bracket Approach to the Dynamics of Nematic Liquid Crystals, Phys. Rev. E 67, 061709 (2003).

[75] N. Rana and P. Perlekar, Coarsening in the Two-Dimensional Incompressible Toner-Tu Equation: Signatures of Turbulence, Phys. Rev. E 102, 032617 (2020).

[76] T. N. Shendruk, K. Thijssen, J. M. Yeomans, and A. Doostmohammadi, Twist-Induced Crossover from TwoDimensional to Three-Dimensional Turbulence in Active Nematics, Phys. Rev. E 98, 010601(R) (2018).

[77] Formally, we could justify this neglect by introducing birth and death so that the concentration becomes a fast variable; see J. Toner, Birth, Death and Flight: A Theory of Malthusian Flocks, Phys. Rev. Lett. 108, 088102 (2012).

[78] In molecular or colloidal systems, $\mu / \rho \gg K / \mu$. Assuming this inequality holds for the systems of interest here as well, $G$ and $\bar{G}$ are uniformly negative.

[79] E. M. Purcell, Life at Low Reynolds Number, Am. J. Phys. 45, 3 (1977).

[80] Groupe d'Etude des Cristaux Liquides Orsay, Dynamics of Fluctuations in Nematic Liquid Crystals, J. Chem. Phys. 51, 816 (1969).

[81] A. M. Leshansky and O. Kenneth, Surface Tank Treading: Propulsion of Purcells Toroidal Swimmer, Phys. Fluids 20, 063104 (2008).

[82] M. Leoni and T. B. Liverpool, Swimmers in Thin Films: From Swarming to Hydrodynamic Instabilities, Phys. Rev. Lett. 105, 238102 (2010).
[83] Even for slow swimmers, one could imagine inertia, in the form of $R$, overcoming the force-dipole instability in the case of a swimmer with a small dipole (and, hence, a small $\sigma_{0}$ ), propelled mainly by its quadrupole force moment.

[84] Spectral Methods in Fluid Dynamics, Springer Series in Computational Physics, edited by C. Canuto, M. Y. Hussaini, A. Quarteroni, and T. A. Zang (Springer, New York, 1988).

[85] S. Cox and P. Matthews, Exponential Time Differencing for Stiff Systems, J. Comput. Phys. 176, 430 (2002).

[86] D. A. Egolf and H. S. Greenside, Characterization of the Transition from Defect to Phase Turbulence, Phys. Rev. Lett. 74, 1751 (1995).

[87] C. Jayaprakash, F. Hayot, and R. Pandit, Universal Properties of the Two-Dimensional Kuramoto-Sivashinsky Equation, Phys. Rev. Lett. 71, 12 (1993).

[88] Such analyses are most useful in the context of an exchange of dominance between system size $L$ and correlation length $\xi$ as one goes from $L<\xi$ to $L>\xi$, with no other scale playing a role. This exchange requires studies at several values of $L$ substantially larger than a short-distance cutoff below which lie the fast modes which contribute the noise actuating the slow modes. For our system, this cutoff scale is the wavelength of the fastestgrowing modes of the unstable linear theory. We defer the required exceedingly large-scale numerical studies to later work.

[89] The value of $\xi$ obtained from the fit is comparable to the one obtained using the definition $\xi \equiv \int_{0}^{\mathcal{L} / 2} C(r) d r$.

[90] R. Alert, J. Casademunt, and J.-F. Joanny, Active turbulence, arXiv:2104.02122.

[91] D. Saintillan and M. J. Shelley, Emergence of Coherent Structures and Large-Scale Flows in Motile Suspensions, J. R. Soc. Interface 9, 571 (2012).

[92] H. Li, X.-Q. Shi, M. Huang, X. Chen, M. Xiao, C. Liu, H. Chaté, and H. P. Zhang, Data-Driven Quantitative Modeling of Bacterial Active Nematics, Proc. Natl. Acad. Sci. U.S.A. 116, 777 (2019).

[93] R. Alert, J. F. Joanny, and J. Casademunt, Universal Scaling of Active Nematic Turbulence, Nat. Phys. 16, 682 (2020).

[94] A. J. Bray, Theory of Phase-Ordering Kinetics, Adv. Phys. 51, 481 (2002).

[95] We verify that the results of our DNS do not change if the advective nonlinear term in Eq. (1) is absent.

[96] L. Smith and W. Reynolds, The Dissipation-Range Spectrum and the Velocity-Derivative Skewness in Turbulent Flows, Phys. Fluids A 3, 992 (1991).

[97] U. Frisch, Turbulence: The Legacy of A. N. Kolmogorov (Cambridge University Press, Cambridge, England, 1995).

[98] G. Duclos, A. Raymond, D. Banerjee, S. Matthew, M. Varghese, I. Kolvin, A. Baskaran, R. Pelcovits, T. Powers, A. Baskaran, F. Toschi, M. Hagan, S. Streichan, V. Vitelli, D. Beller, and Z. Dogic, Topological Structure and Dynamics of Three-Dimensional Active Nematics, Science 367, 1120 (2020).

[99] Z. Krajnik, Z. Kos, and M. Ravnik, Spectral Energy Analysis of Bulk Three-Dimensional Active Nematic Turbulence, Soft Matter 16, 9059 (2020). 
[100] A. Lau and T. C. Lubensky, Fluctuating Hydrodynamics and Microrheology of a Dilute Suspension of Swimming Bacteria, Phys. Rev. E 80, 011917 (2009).

[101] S. R. acknowledges a discussion with Ignacio Pagonabarraga Mora on this point.

[102] P. T. Underhill, J. P. Hernandez-Ortiz, and M. D. Graham, Diffusion and Spatial Correlations in Suspensions of Swimming Particles, Phys. Rev. Lett. 100, 248101 (2008).

[103] G. F. Mazenko, Nonequilibrium Statistical Mechanics (Wiley-VCH Verlag, Berlin, 2006).

[104] D. Forster, Hydrodynamic Fluctuations, Broken Symmetry, and Correlation Functions, Frontiers in Physics: A Lecture Note and Reprint Series (Benjamin, New York, 1975).
[105] Y. Kuramoto and T. Tsuzuki, Persistent Propagation of Concentration Waves in Dissipative Media far from Thermal Equilibrium, Prog. Theor. Phys. 55, 356 (1976).

[106] G. Sivashinsky, On Self-Turbulization of a Laminar Flame, Acta Astronaut. 6, 569 (1979).

[107] T. Dombrowski, S. K. Jones, G. Katsikis, A. P. S. Bhalla, B. E. Griffith, and D. Klotsa, Transition in Swimming Direction in a Model Self-Propelled Inertial Swimmer, Phys. Rev. Fluids 4, 021101(R) (2019).

[108] T. Dombrowski and D. Klotsa, Kinematics of a Simple Reciprocal Model Swimmer at Intermediate Reynolds Numbers, Phys. Rev. Fluids 5, 063103 (2020). 